\title{
Aproximación teÓRICA a LAS bases de UNA ESTRATEgIA TECNOLÓGICA ORIENTADA A LA REDUCCIÓN DEL RIESGO DE desastres en América Latina y el Caribe ${ }^{1}$
}

\author{
Alberto Alejandro Iberico Hervias \\ aibericoh@gmail.com
}

\begin{abstract}
Resumen
América Latina y el Caribe constituyen una región expuesta a múltiples amenazas originadas por la naturaleza y la actividad humana, las cuales combinadas con las vulnerabilidades socioeconómicas, políticas y ambientales existentes, traen como resultado la ocurrencia de desastres de gran magnitud que afectan el proceso del desarrollo humano y sostenible de las sociedades. Mientras que el Marco de Acción de Hyogo (MAH) representa un acuerdo global orientado a reducir considerablemente las pérdidas humanas, económicas y ambientales ocasionadas por los desastres; el Plan de Acción Regional eLAC es una estrategia que considera a las tecnologías de la información y de la comunicación (TIC) como instrumentos promotores del desarrollo y la inclusión social en el contexto de la sociedad de la información. En base al análisis de la convergencia entre ambos esfuerzos, el presente artículo desarrolla una aproximación teórica a las bases de una estrategia tecnológica, la cual reorienta, a través de un conjunto de lineamientos, las metas del Plan de Acción Regional eLAC hacia la consecución de los objetivos y prioridades de acción del Marco de Acción de Hyogo. Por consiguiente, se considera que el planteamiento de esta estrategia establece los cimientos necesarios, que, una vez puestos en práctica bajo un verdadero compromiso institucional, contribuirán en la reducción del riesgo de desastres en la región.
\end{abstract}

Palabras clave: Reducción del Riesgo de Desastres, Sociedad de la Información, Marco de Acción de Hyogo, Plan de Acción Regional eLAC, Tecnologías de la Información y las Comunicaciones, Desarrollo humano, Estrategia Tecnológica.

1 El presente documento está basado en un trabajo de investigación elaborado y sustentado en el marco del Máster en Cooperación Internacional: Paz y Desarrollo impartido en la Facultad de Ciencias Sociales y de la Comunicación, Departamento de Derecho Internacional Público, Relaciones Internacionales e Historia del Derecho, de la Universidad del País Vasco. El propósito de esta investigación fue compartir una iniciativa sobre cómo las tecnologías de la información y las comunicaciones pueden contribuir estratégicamente a la reducción del riesgo de desastres, la cual representa un área clave dentro del campo del desarrollo. 
En los últimos años se ha evidenciado, en distintos países y regiones del mundo, cuantiosas pérdidas humanas, económicas y ambientales por la ocurrencia de desastres originados por la naturaleza y la acción del hombre. En la coyuntura actual, uno de los posibles factores del incremento de dichos desastres es el cambio climático y su relación con las distintas vulnerabilidades existentes en los estados. Las recientes pérdidas a nivel global causadas por los impactos de los desastres demuestran la necesidad imperante de los gobiernos de incorporar y fortalecer en sus políticas, estrategias y planes de desarrollo, los procesos de la Reducción del Riesgo de Desastres (RRD) y la adaptación al cambio climático. Solamente en el año 2010, las pérdidas ascendieron a más de 297 mil muertes y 217 millones de personas afectadas, dejando un saldo de 123,9 billones de dólares en daños económicos (Guha-Sapir et al. 2011). ${ }^{2}$

Con los lamentables antecedentes del terremoto, tsunami y posterior crisis nuclear que azotaron el nordeste de Japón en marzo de 2011, ${ }^{3}$ se realizó la tercera reunión de la Plataforma Global para la Reducción del Riesgo de Desastres (PG/ RRD), que se celebró en mayo del 2011 en Ginebra, Suiza (EIRD 2011a). Esta conferencia, auspiciada por la Estrategia Internacional para la Reducción de Desastres (EIRD), representó la reafirmación del compromiso y seguimiento del progreso en la implementación del Marco de Acción de Hyogo por parte de los líderes de los gobiernos, organismos internacionales, expertos técnicos y sociedad civil. Este marco, cuya vigencia continúa hasta el 2015, representa una estrategia internacional orientada a la Reducción del Riesgo de Desastres ${ }^{4} y$ al aumento de la Resiliencia ${ }^{5}$ de las naciones en pos del desarrollo sostenible.

En el ámbito de nuestro continente, América Latina y el Caribe constituyen una región expuesta a múltiples y recurrentes amenazas naturales, entre las cuales se encuentran huracanes, lluvias, terremotos, deslizamientos de tierra, erupciones volcánicas, sequías, inundaciones, heladas, entre muchas otras amenazas.

2 En términos económicos a nivel global, entre los desastres más importantes del 2010 se encuentran los terremotos de Haití, Chile y Nueva Zelanda; las inundaciones en Pakistán, Tailandia y Australia; y las tormentas de nieve ocurridas en varios países de Europa (Guha-Sapir et al. 2011).

3 Se calcula que las cifras del desastre generado por el terremoto y el tsunami en Japón el 11 de marzo del 2011 ascienden a 28.050 muertes y 309 billones de dólares en daños económicos (CRED 2011).

4 La reducción del riesgo de desastres representa un marco conceptual de elementos que tienen la función de minimizar las vulnerabilidades y los riesgos en una sociedad, para evitar (prevención) o limitar (mitigación y preparación) el impacto adverso de las amenazas, dentro del amplio contexto del desarrollo sostenible (EIRD 2004).

5 La resiliencia se define como la capacidad de un sistema, comunidad o sociedad potencialmente expuestas a amenazas a adaptarse, resistiendo o cambiando con el fin de alcanzar y mantener un nivel aceptable en su funcionamiento y estructura (EIRD 2004). 
Paralelamente, las estructuras socioeconómicas, políticas, culturales y ambientales de la mayoría de los países latinoamericanos han generado estructuralmente un conjunto de vulnerabilidades entre las cuales se encuentran los débiles marcos institucionales y normativos de los gobiernos; los altos niveles de pobreza y desigualdad; la falta de acceso a los servicios básicos, como son salud, educación y saneamiento; el desordenado crecimiento de las ciudades; el aumento exponencial de la población; el debilitamiento de los ecosistemas y la degradación ambiental.

La combinación de estas amenazas, ya sean de origen natural o propiciadas por el hombre, con las vulnerabilidades estructurales de los países de la región, da como resultado la ocurrencia de los desastres. Estos eventos representan graves interrupciones en el funcionamiento de las comunidades o sociedades que son traducidas en impactos o pérdidas sociales, económicas y ambientales (EIRD 2004). Un claro ejemplo de ello en la región se da partir de la comparación del impacto ocasionado por los terremotos de Haití y Chile en 2010, donde se evidencia que el país caribeño sufrió mayores pérdidas en términos de vidas humanas debido a sus muy acentuadas vulnerabilidades. ${ }^{6}$ Esto sucedió a pesar de que, según la escala de Richter, el terremoto de Chile tuvo una mayor liberación de energía.

Es entonces que los desastres interrumpen seriamente el proceso de desarrollo de las sociedades, y los países en vías de desarrollo son los más afectados dadas sus altas condiciones de vulnerabilidad, sus débiles capacidades de respuesta y la carencia de una efectiva resiliencia. En ese sentido, uno de los grandes retos en la región es lograr, en forma conjunta y bajo responsabilidad de cada Estado, la reducción de los desastres a fin de garantizar la continuidad del desarrollo de los países. De esta manera, el MAH representa una guía esencial para gestionar y reducir el riesgo en los ámbitos regional, nacional, local y comunitario; incorporando objetivos y medidas para minimizar las vulnerabilidades existentes y las que posiblemente se generen; previniendo y afrontando las amenazas naturales o causadas por el hombre; fortaleciendo los mecanismos de preparación, respuesta y reconstrucción ante desastres; aumentando las capacidades y la resiliencia a través de una cultura de prevención y participación conjunta; y focalizando la urgente atención a los grupos sociales más vulnerables.

Ante la necesidad de dar cumplimiento a la agenda global en materia de reducción del riesgo de desastres, las TIC tienen un rol estratégico e importante.

6 Considerado uno de los países más pobres del mundo, Haití posee un déficit en el acceso a servicios básicos de salud y educación, junto con una historia de años de conflictos, inestabilidad política y recurrencia de desastres. La combinación de estas vulnerabilidades estructurales y la ocurrencia del terremoto de enero de 2010 dieron como resultado la muerte de 222.570 personas y una pérdida económica que ascendió a 8 billones de dólares, sobrepasando su PBI (Guha-Sapir et al. 2011). 
Esto encuentra sustento en que la gestión de la información es un factor crucial en el desarrollo y fortalecimiento de políticas, estrategias y procesos de reducción del riesgo, sobre todo en las etapas concernientes a la identificación del riesgo, la preparación y la respuesta. A partir de que las TIC son consideradas instrumentos transversales para el apoyo de las áreas claves del desarrollo, es conveniente orientar estratégicamente su aplicación en la implementación de los objetivos y prioridades definidas en el Marco de Acción de Hyogo.

Al concebir las TIC como instrumentos para la búsqueda de un desarrollo humano ${ }^{7}$ más equitativo y sostenible, y considerar el desarrollo prioritario de la sociedad de la información ${ }^{8}$ en la región, el Plan de Acción Regional sobre la Sociedad de la Información en América Latina y el Caribe (eLAC) representa una estrategia concertada que busca lograr, a través de políticas y lineamientos tecnológicos, un mayor crecimiento económico y una adecuada inclusión social en el ámbito digital. La Tercera Conferencia Ministerial sobre la Sociedad de la Información de América Latina y el Caribe, celebrada en Lima, en noviembre del 2010, estableció el inicio de la tercera etapa de esta estrategia denominada Plan eLAC2015. Esta conferencia, auspiciada por la Comisión Económica para América Latina y el Caribe (Cepal), representó una vez más la voluntad regional para alcanzar una agenda compartida en pos del desarrollo de las sociedades de la información de Latinoamérica (Cepal 2011a).

Por lo tanto, partiendo del panorama actual de la región en el cual se busca el fortalecimiento de la reducción del riesgo de desastres, y tomando como referencia los avances ya iniciados hacia el desarrollo de la sociedad de la información, el presente artículo esboza una aproximación a las bases de una estrategia tecnológica que propone canalizar, a través de un conjunto de lineamientos, las metas del Plan de Acción Regional eLAC hacia la consecución de los objetivos y prioridades del Marco de Acción de Hyogo. Este planteamiento inicial establece la forma como las TIC pueden contribuir a la implementación de políticas, estrategias y procesos de reducción del riesgo a nivel institucional con el fin de asegurar la continuidad del desarrollo de los países de América Latina y el Caribe.

7 En el documento Informe sobre Desarrollo Humano 1990 se le define como un proceso mediante el cual se ofrece a las personas la libertad y la ampliación de escogencia de oportunidades para lograr un determinado bienestar y calidad de vida por medio del desarrollo de sus capacidades (PNUD 1990).

8 Existen muchos conceptos que intentan definir la sociedad de la información. Manuel Castells utiliza el término sociedad red para referirse a este proceso definiéndolo como "el tipo de organización social resultante de la interacción entre, por un lado, la revolución tecnológica basada en la digitalización electrónica de la información y la comunicación, y la ingeniería genética y por otro lado, los procesos sociales, económicos, culturales y políticos del último cuarto del siglo XX" (Castells 1997). 
Cabe señalar que esta estrategia determina el modo como deben ser gestionadas las TIC, preparando el terreno para su posterior aplicación en los niveles operacional y técnico. Asimismo, la estrategia tecnológica se fundamenta en la convergencia del MAH y el eLAC, esfuerzos que se encuentran alineados con el cumplimiento de los objetivos de desarrollo del milenio en el horizonte 2015 (ONU 2011); compartiendo características similares en su estructura y organización; y reconociendo el carácter crucial de la gestión de la información en los procesos de la reducción del riesgo a través del uso de las tecnologías.

\section{Una guía internacional para la reducción del riesgo de} desastres: El marco de acción de Hyogo

El Marco de Acción de Hyogo 2005-2015: Aumento de la Resiliencia de las Naciones y las Comunidades ante los Desastres fue adoptado por 168 estados durante la segunda Conferencia Mundial sobre la Reducción de Desastres (CMRD), celebrada entre el 18 y el 22 de enero del 2005 en Kobe, prefectura de Hyogo, Japón. ${ }^{9}$ Este acuerdo internacional surgió ante la necesidad de contar con una estrategia global de reducción de desastres en el horizonte 2005-2015 y como consecuencia de la sucesión de numerosos desastres de gran escala ocurridos durante los primeros años de la década del $2000^{10}$ (ONU 2005).

Cabe señalar que este consenso también fue resultado de un proceso de evolución institucional a través de distintos acuerdos y compromisos internacionales que buscaron reducir el impacto de los desastres. Entre los más importantes destacan el Decenio Internacional para la Reducción de Desastres Naturales (DIRDN), creado en 1989; la Estrategia y Plan de Acción de Yokohama para un mundo más seguro, acordado en 1994; la Estrategia Internacional para la Reducción del Riesgo de Desastres creada en 1999; y la Cumbre Mundial sobre el Desarrollo Sostenible (CMDS), celebrada en Johannesburgo, Sudáfrica, el 2002.

Con el propósito de incrementar el compromiso y la acción conjunta en la implementación de este marco, la Plataforma Global para la Reducción del Riesgo

9 La conferencia representó una gran oportunidad para fomentar un enfoque estratégico y sistemático para la reducción del riesgo de desastres. Asimismo, puso de manifiesto la necesidad y el camino para lograr aumentar la resiliencia de las naciones y las comunidades ante los desastres (ONU 2005).

10 Durante la conferencia se aprobó la Declaración de Hyogo, la cual representó un conjunto de premisas y declaraciones hechas por los delegados de la CMRD con el fin de promover el compromiso global de reducir las pérdidas causadas por los desastres. Asimismo, cabe destacar la Declaración Común de la Reunión Especial sobre el Desastres el Océano Índico: Reducción de los Riesgos para un Futuro más Seguro, la cual fue constituida como consecuencia del gran impacto causado por el tsunami del océano Índico en diciembre del 2004 (ONU 2005). 
de Desastres fue establecida como el primer foro consultivo para la reducción del riesgo a nivel global. Esta plataforma es celebrada cada dos años en la sede de la secretaría de la EIRD en Ginebra, Suiza, y su objetivo principal es garantizar la coyuntura favorable a escala mundial para promover la resiliencia de las naciones y comunidades a través del Marco de Acción de Hyogo"1 (EIRD 2010).

De esta manera, el horizonte de trabajo que estableció la PG/RRD comenzó en junio del 2007, a través de una primera sesión que fue mayoritariamente percibida por los participantes como un importante hito en el movimiento mundial para la reducción del riesgo de desastres. Posteriormente, la segunda sesión fue celebrada en junio del 2009, en el contexto de la creciente alarma por el cambio climático e incremento del riesgo. En esta reunión, los representantes de los estados destacaron que la reducción del riesgo es un elemento crítico para la gestión de los efectos del cambio climático y para la reducción del deterioro del bienestar social y económico (EIRD 2009).

Recientemente fue celebrada la "Tercera Sesión de la Plataforma Global para la Reducción del Riesgo de Desastres: Invertir hoy para un mañana más seguro, una mayor inversión en medidas locales", la cual estableció como objetivos principales dar a conocer los resultados de la evaluación de la mitad del progreso del Marco de Acción de Hyogo; abordar los temas sobre la reconstrucción y recuperación, la economía de la reducción del riesgo de desastres, y las alianzas para el cambio climático y el desarrollo; y celebrar en forma paralela la Conferencia Mundial sobre Reconstrucción (EIRD 2011a).

Cabe señalar que en el ámbito regional existen distintos esfuerzos institucionales vigentes que han adoptado el Marco de Acción de Hyogo, entre los cuales destacan el Caribbean Disaster Emergency Management Agency (CDEMA), adoptado en 1991 por la subregión del Caribe y actualizado posteriormente en el 2009; el Centro de Coordinación para la Prevención de Desastres Naturales en América Central (Cepredenac), creado por los países de Centroamérica en 1988; y el Comité Andino para la Prevención y Atención de los Desastres (Caprade), acordado en el 2002 por los países miembros de la Comunidad Andina (EIRD 2011b).

11 Entre las funciones principales de esta plataforma destacan la evaluación del progreso en la implementación del MAH; el incremento del conocimiento en materia de RRD; el intercambio de experiencias y aprendizaje de buenas prácticas; y la identificación de las acciones necesarias para acelerar la ejecución del MAH a escalas nacional y local (ONU 2008: 4) 


\subsection{Descripción del marco}

El MAH establece un conjunto de objetivos y prioridades de acción que los estados y actores relevantes ${ }^{12}$ deben alcanzar para el año 2015 como consecución de los Objetivos de Desarrollo del Milenio. Considerando que el MAH no tiene carácter vinculante desde el punto de vista jurídico, los gobiernos y actores relevantes tienen la facultad de decidir la forma de su implementación en función de sus necesidades y capacidades ${ }^{13}$ (EIRD 2007a). En el cuadro 1 se muestra el resumen de este marco.

Resumen del Marco de Acción de Hyogo para el 2005-2015: Aumento de la Resiliencia de las Naciones y las Comunidades ante los Desastres (Marco de Hyogo)

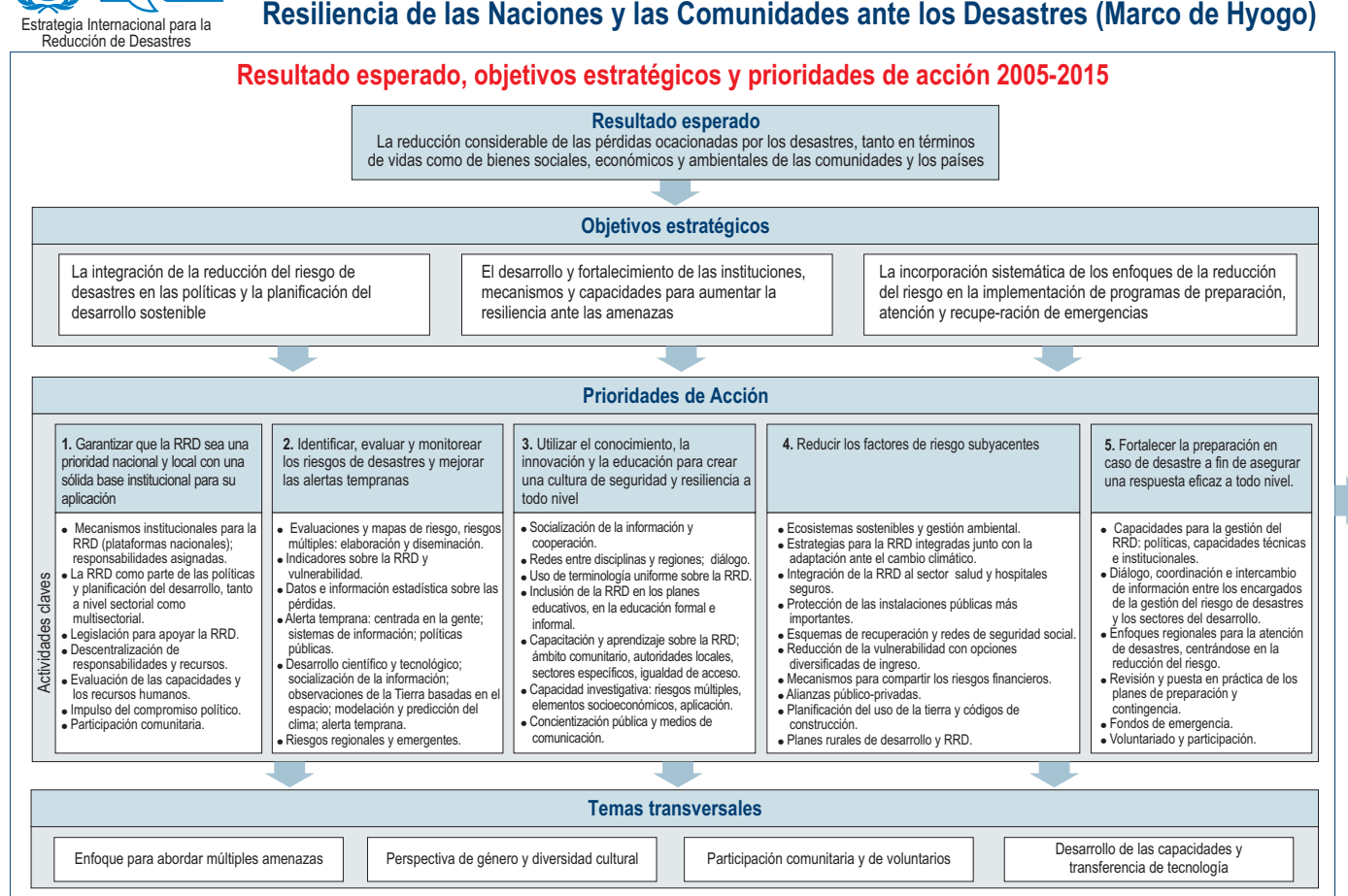

Cuadro 1: Resumen del MAH para el 2005-2015

Fuente: EIRD (2007a).

12 Como actores relevantes se encuentran las organizaciones regionales e internacionales, la sociedad civil, el sector privado y la comunidad científica.

13 El MAH fue el resultado de un profundo estudio sobre las tendencias en los riesgos de desastre y sobre las experiencias que se han venido generando en cuanto a este tema a lo largo de los años. Para su elaboración se llevaron a cabo intensas negociaciones a lo largo del 2004 y a principios del 2005 (EIRD 2007a). 
De acuerdo con esto, el objetivo general del MAH es reducir considerablemente las pérdidas ocasionadas por los desastres, tanto en términos de vidas humanas como de bienes sociales, económicos y ambientales de las comunidades y los países. A fin de lograr este cumplimiento, el marco define tres objetivos estratégicos que sirven de bases para el desarrollo de las prioridades de acción (ONU 2005):

- Primer objetivo estratégico.- Integrar la reducción del riesgo de desastres en las políticas, planes y programas de desarrollo sostenible a todo nivel.

- Segundo objetivo estratégico.- Desarrollar y fortalecer las instituciones, mecanismos y capacidades que permitan aumentar la resiliencia ante las amenazas.

- Tercer objetivo estratégico-- Incorporar sistemáticamente los criterios de la reducción del riesgo de desastres en la implementación de programas de preparación, atención y recuperación de emergencias.

A partir de esto, las prioridades de acción del MAH constituyen un conjunto de actividades que deben ser ejecutadas por los gobiernos nacionales en conjunto con los grupos multisectoriales, los gobiernos locales, las comunidades y los actores relevantes a fin de implementar la reducción del riesgo a todo nivel. ${ }^{14} \mathrm{~A}$ continuación se realiza un breve resumen de cada prioridad:

- Prioridad 1.- Garantizar que la reducción del riesgo de desastres sea una prioridad nacional y local con una sólida base institucional para su implementación. Esta primera prioridad establece las medidas para propiciar un espacio de diálogo entre los grupos multisectoriales interesados en aplicar los fundamentos de la reducción del riesgo. Asimismo, promueve la creación y el desarrollo de los mecanismos de coordinación nacionales, conocidos como plataformas nacionales, las cuales facilitan la ejecución de acciones coordinadas y efectivas respecto a la reducción del riesgo de desastres. Como punto importante, se fomenta el desarrollo de una institucionalidad sustentada en un marco político y legal adecuado. Por último, se promueve la integración de la reducción del riesgo de desastres en los planes de desarrollo y la asignación de recursos para el cumplimiento efectivo de estos planes (EIRD 2007a).

- Prioridad 2.-Identificar, evaluar y monitorear los riesgos de desastre y mejorar las alertas tempranas.

La segunda prioridad establece un conjunto de iniciativas para realizar una evaluación del riesgo de todo un ámbito nacional. Sumado a esto, se propone la revisión de la disponibilidad de la información crítica sobre riesgos, la capaci-

14 El establecimiento de las cinco prioridades de acción son el resultado de las conclusiones del examen de la estrategia y plan de acción de Yokohama para un mundo más seguro y de las deliberaciones de la Cumbre Mundial sobre el Desarrollo Sostenible (ONU 2005). 
dad para la captura de datos y su óptima utilización. Además, se establece la necesidad de evaluar las capacidades y fortalecer los sistemas de alerta temprana centrados en las personas. Finalmente, se promueve el desarrollo de mecanismos de comunicación y difusión de la alerta temprana con el propósito de contar con información confiable sobre el riesgo de desastres (EIRD 2007a).

- Prioridad 3.- Utilizar el conocimiento, la innovación y la educación para crear una cultura de seguridad y resiliencia en todos los niveles.

En esta prioridad se fomenta el desarrollo de programas de concientización sobre la reducción del riesgo. Asimismo, se establece el requerimiento de incorporar la reducción del riesgo de desastres en el sistema educativo nacional y en las comunidades de investigación científica. También se propone la puesta en marcha de programas de capacitación en la reducción de desastres en los sectores clave de desarrollo, en los ámbitos comunitario y local, y para los grupos más vulnerables. Por último, se promueve la mejora del proceso de gestión de la información para el uso de los gobiernos nacionales, locales, comunidades y actores relevantes (EIRD 2007a).

- Prioridad 4.- Reducir los factores de riesgo subyacentes.

La cuarta prioridad se centra en la reducción del riesgo en los sectores del desarrollo más importantes. De acuerdo con esto, se promueve la incorporación de la reducción del riesgo de desastres en la gestión ambiental y en uso de los recursos naturales. De igual forma, se establecen los mecanismos para aumentar la resiliencia de los grupos sociales en situación de pobreza y vulnerabilidad. Se promueven medidas para incorporar la reducción del riesgo en la planificación urbana y en el ordenamiento territorial, así como también para garantizar la seguridad y la protección de las instalaciones críticas, públicas y privadas. Por otro lado, se propone estimular las actividades de reducción de desastres en los sectores de producción y servicios, creando oportunidades de participación por parte del sector privado. Por último, se requiere el desarrollo de un proceso de planificación para la recuperación posdesastre que incorpore necesariamente la reducción del riesgo (EIRD 2007a).

- Prioridad 5.- Fortalecer la preparación ante los desastres para dar una respuesta eficaz en todos los niveles.

Considerando que la quinta prioridad se encuentra focalizada a la consecución del tercer objetivo estratégico del marco, esta propicia un entendimiento común entre los distintos actores y actividades que contribuyen a la preparación y gestión de emergencias. Asimismo, se promueve la evaluación de las capacidades y mecanismos para contar con una respuesta eficaz ante dichos eventos. Por último, se establecen las medidas necesarias para fortalecer la planificación de la preparación ante desastres por medio de la mejora de los planes de preparación, contingencia, respuesta y recuperación (EIRD 2007a). 
Una vez descritas estas prioridades, cabe indicar que el MAH establece un conjunto de responsabilidades para los estados; las organizaciones regionales e internacionales; y para la estrategia internacional para la reducción de desastres; con el fin de garantizar su implementación e incrementar la resiliencia de las naciones. Por consiguiente, la máxima responsabilidad recae en los gobiernos, cuyas medidas sobre la Reducción del Riesgo de Desastres deben ser orientadas a todo nivel: nacional, local y comunitario, sobre todo para los grupos más vulnerables. No obstante, es importante contar con la cooperación de las entidades regionales e internacionales especializadas a través de la transferencia de conocimientos y asistencia técnica para los países en vías de desarrollo, sobre todo a los más propensos a sufrir desastres (ONU 2005).

De igual manera, se destaca las responsabilidades del sistema EIRD, las cuales consisten en apoyar las políticas y mecanismos de coordinación nacionales, fomentar la coordinación regional e internacional, estimular el intercambio de información y buenas prácticas, garantizar la colaboración de las agencias de las Naciones Unidas ${ }^{15}$ y otras organizaciones, y evaluar con periodicidad el progreso de la implementación del MAH (EIRD 2007a).

\section{Una estrategia regional para el desarrollo de la sociedad de la} información: El plan de acción regional ELAC

Ante la necesidad global de abordar los temas inherentes al proceso establecido por la Sociedad de la Información, y a la exclusión social y económica que ha generado el fenómeno de la brecha digital, la Cumbre Mundial sobre la Sociedad de la Información, (CMSI) representó una serie de reuniones en las cuales uno de los desafíos planteados más importante fue el encauzamiento del potencial de las TIC a la consecución y promoción de los Objetivos del Desarrollo del Milenio ${ }^{16}$ (CSMI 2005). Esta cumbre estuvo constituida en dos fases, la primera llevada a cabo en Ginebra en diciembre del 2003, y la segunda en Túnez, en noviembre del 2005.7

15 Entre las agencias de las Naciones Unidas que poseen un mandato específico y un compromiso importante en la reducción del riesgo de desastre se encuentran el PNUD, la OMM, el PNUMA, la Unesco, la ONU/Habitat, la Unicef y la OCAH (EIRD 2007b).

16 En la actualidad, las TIC son consideradas herramientas estratégicas para promover el desarrollo humano. Esto se basa en el hecho de que la información y el conocimiento son recursos esenciales para ampliar las opciones humanas y mejorar la calidad de vida, tanto a nivel individual como colectivo (Acevedo et al. 2006).

17 La Unión Internacional de Telecomunicaciones (UIT), como representante de las Naciones Unidas y organismo especializado, ha sido la encargada de gestionar el proceso de la CMSI y sus posteriores eventos, los cuales continúan vigentes en la actualidad. Asimismo, se destaca la participación que tuvieron los gobiernos nacionales, organizaciones internacionales, la sociedad civil y representantes del sector privado. 
Considerando la importancia de implementar los principios y metas acordados en este consenso internacional, las autoridades de los gobiernos de América Latina y el Caribe decidieron intensificar sus esfuerzos hacia la adopción de una visión regional concertada que buscaba el desarrollo de las sociedades de la información..$^{18} \mathrm{~A}$ través de diversos acuerdos y reuniones de preparación, estos esfuerzos fueron consolidados en el Compromiso de Río, en el cual se constituyó el Plan de Acción Regional sobre la Sociedad de la Información en América Latina y el Caribe. Este hecho fue celebrado en la Conferencia Regional Ministerial de América Latina y el Caribe preparatoria para la segunda fase de la CMSI, celebrada en Río de Janeiro en junio del 2005 (Cepal 2010c).

\subsection{Descripción del plan de acción}

El Plan de Acción Regional eLAC fue creado como una estrategia concertada a nivel regional, la cual considera principalmente a las TIC como instrumentos promotores del desarrollo económico e inclusión social en los países ${ }^{19}$ (CEPAL, 2010b). Así mismo, esta estrategia representa una plataforma que promueve la integración regional y la cooperación en materia tecnológica, actuando como articulador de las metas a nivel internacional, y las necesidades y prioridades de los países de América Latina y el Caribe (CEPAL, 2010a). Cabe señalar que la Comisión Económica para América Latina y el Caribe, CEPAL, cumple la función de secretaría técnica en pos del cumplimiento de este plan de acción. De acuerdo a esto, en el Cuadro $N^{\circ} 2$ se muestra la hoja de ruta del Plan de Acción Regional eLAC para el periodo 2005-2015.

18 Previo a esto, ya existía la necesidad por parte de los gobiernos de incorporar estrategias de inclusión digital o estrategias digitales en sus políticas y planes de desarrollo, buscando de esta manera potenciar a través de las TIC todas las esferas del desarrollo (Cepal 2009).

19 En el Compromiso de San Salvador los países de la región afirmaron como visión "la determinación común de construir una sociedad de la información integradora orientada al desarrollo y centrada en la persona, basada en los principios de paz, derechos humanos, solidaridad, libertad, democracia, desarrollo sostenible y cooperación, para que los seres humanos, las comunidades y los pueblos de América Latina y el Caribe pueden aprovechar plenamente sus potencialidades en la promoción de su desarrollo" (Cepal 2010a). 


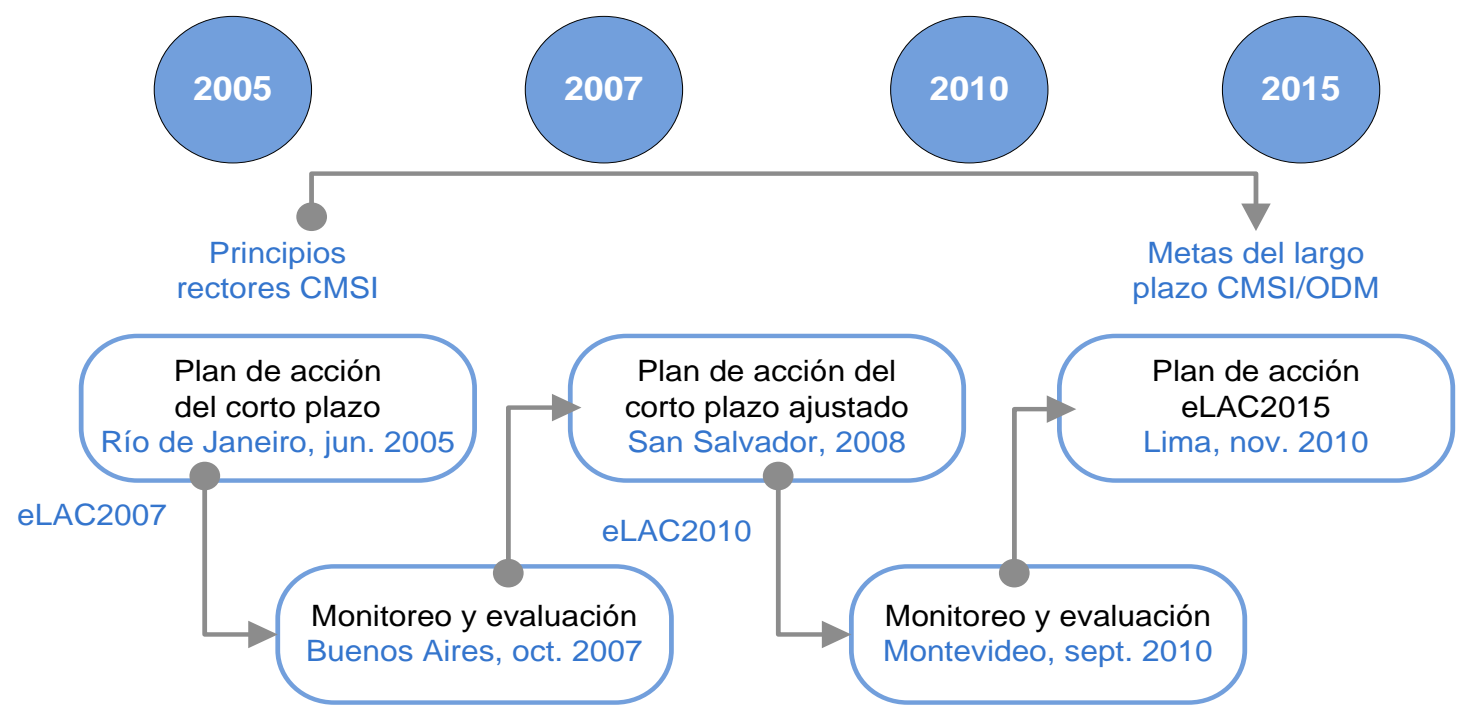

Cuadro 2. El proceso eLAC 2005-2015, una visión a largo plazo con planes de acción a corto plazo.

Fuente: Cepal (2010d).

Es entonces que la primera etapa de esta estrategia, Plan eLAC2007, tuvo como principales objetivos el progreso hacia una sociedad de la información que beneficie a los habitantes de la región, el fomento del desarrollo económico y social, la consolidación de la democracia y el fortalecimiento de la integración regional, y la aceleración de este progreso mediante la reducción de costos económicos y sociales ${ }^{20}$ (Cepal 2010c). Para la segunda fase, el Plan eLAC2010 representó una mejora y renovación del Plan eLAC2007, e incluyó una serie de modificaciones importantes a las prioridades de la región en cuanto al acceso, uso y capacidades relacionadas a las TIC.

El Plan eLAC2015,21 acordado para el periodo 2011-2015, representa la tercera y última etapa de esta estrategia regional en su horizonte 2015. Aquí se incorpora por primera vez, dentro de sus principales lineamientos, el tema del medioambiente.

20 El contenido del Plan de Acción eLAC2007 se encuentra en el documento Plan de Acción Regional sobre la Sociedad de la Información de América Latina y el Caribe eLAC2007 (Cepal 2007). Tal como fue mencionado, este Plan fue constituido en el Compromiso de Río de Janeiro, en junio del 2005.

21 I detalle del Plan de Acción eLAC2015 se encuentra en el documento Plan de Acción sobre la Sociedad de la Información y del Conocimiento de América Latina y el Caribe (eLAC2015) (Cepal 2011b). Tal como fue mencionado, este plan fue constituido en la Declaración de Lima, en noviembre del 2010 
De acuerdo con esto, se establece la promoción del uso de las TIC para la mitigación del impacto climático, ${ }^{22}$ y la prevención y atención de los desastres naturales o situaciones de emergencia. Asimismo, el eLAC2015 enfatiza el desafío de fortalecer los planes de emergencia y seguridad ante los riesgos de desastres a través del uso de sistemas de información y alerta temprana a la ciudadanía, las redes de comunicaciones robustas para el gobierno, y los servicios de telecomunicaciones confiables para que las personas puedan comunicarse entre sí por medio de celulares, computadoras o televisión digital interactiva abierta ${ }^{23}$ (Cepal 2011 b).

Cabe señalar que para propósitos del presente estudio se han tomado como referencia los lineamientos y metas del Plan eLAC2010. Dicho plan, cuyo periodo comprendió entre el 2008 y el 2010, estuvo conformado por un total de 83 metas agrupadas en 6 capítulos, de las cuales 18 estaban orientadas a resultados cuantificables y 65 para acciones no cuantificables. ${ }^{24}$ A continuación se procede a resumir este conjunto de metas:

- Capítulo 1. Educación.- En este primer capítulo se detallan el desarrollo de programas de estudio de gestión de datos, información y conocimiento; y la elaboración de estudios anuales sobre el impacto del uso de las TIC en el sistema educativo. De igual forma, se promueven el acceso a Internet para las instituciones de enseñanza pública a través de la banda ancha; y el aumento de las capacidades por medio de la preparación de los estudiantes, profesores y funcionarios públicos del sector educación. Sumado a esto, se fomenta el desarrollo de aplicaciones orientadas a la educación, tales como los portales educativos, las herramientas de realidad virtual ${ }^{25}$ y los servicios de contenido difundidos por las redes educativas regionales (Cepal 2010a).

22 Considerando que las emisiones de CO2 contribuyen al calentamiento global, el Plan eLAC2015 hace referencia a la huella de carbono de las TIC (computadoras, periféricos, redes de telecomunicaciones centros de datos y dispositivos), las cuales ascendieron a $830 \mathrm{MtCO} 2$ en el 2007, representando alrededor del $2 \%$ del total de emisiones anuales generadas por actividades industriales. Se proyecta que este porcentaje aumentaría en $6 \%$ para el año 2020. Adicionalmente, se establece una meta que busca fomentar políticas públicas en la gestión integral de los desechos derivados de las TIC y su uso (Cepal 2011b).

23 Otro tema fundamental que promueve el Plan de Acción eLAC2015 es el desarrollo y la universalización del acceso de la Banda Ancha en los países de la región. De esta manera, se considera que esta tecnología referente del siglo XXI tiene la misma importancia para el crecimiento y la igualdad como la tuvieron la infraestructura eléctrica y las carreteras en el siglo XX (Cepal 2011b).

24 El contenido del Plan de Acción eLAC2010 (metas y capítulos) se encuentra en el documento Compromiso de San Salvador (Cepal 2010a). Este plan fue establecido en el Compromiso de San Salvador en el contexto de la Segunda Conferencia Ministerial sobre la Sociedad de la Información de América Latina y el Caribe celebrada en El Salvador, en febrero del 2005.

25 Se especifica que el uso de estas herramientas de realidad virtual educativas tienen como fin fomentar la diversidad cultural, la tolerancia y combatir la discriminación por consideraciones de razas, género, etnia, enfermedad o discapacidades (Cepal 2010a). 
- Capítulo 2. Acceso e infraestructura.- Entre las metas más importantes del segundo capítulo se encuentran la promoción de las TIC para la real inserción social, educativa, cultural y económica de todos los sectores sociales, especialmente para los grupos más vulnerables; la potenciación y apoyo de iniciativas de redes basadas en comunidades que incluyan el uso de tecnologías tradicionales y nuevas, ${ }^{26}$ el apoyo a iniciativas regionales y subregionales que aprovechan las economías de escala para atender a los sectores que se han visto marginados por los avances tecnológicos; y el impulso del desarrollo de la infraestructura en cada país y en la región con el fin de mejorar la calidad de la red y reducir los costos de acceso (Cepal 2010a).

Asimismo, se promueve la cobertura de una red confiable y de alta capacidad en las zonas urbanas y rurales, incrementando los centros de acceso a las TIC al servicio de la comunidad; la elaboración de estudios sobre la estructura de precios de los servicios de telecomunicaciones de telefonía fija, móvil e internet; el impulso de creación de canastas de servicios digitales a precios especiales orientados a los sectores socialmente vulnerables; y la revisión de la funcionalidad, el diseño y objeto de los fondos de acceso universal a las TIC. Por último, particularmente en este capítulo, se destaca el fortalecimiento de la gestión de desastres a través de las TIC, considerando sus fases y requerimientos (Cepal 2010a).

- Capítulo 3. Salud.- En este tercer capítulo se promueve la integración adecuada de las TIC en el sector salud a través del fomento de políticas públicas centradas en las personas y la continuidad de los servicios, el establecimiento de servicios de salud electrónicos basados en Internet en los centros de salud y hospitales públicos; la capacitación de profesionales y tomadores de decisiones del sector salud en el uso de las TIC; y por último, la necesidad de los centros de salud y hospitales para trabajar con aplicaciones para la gestión y planificación de procesos, que estén enlazados a portales nacionales de salud, con miras a mejorar y a la promoción de redes regionales que permitan compartir experiencias y conocimientos (Cepal 2010a).

- Capítulo 4. Gestión pública.- Aquí se da relevancia al fortalecimiento de los servicios de gobierno electrónico a través de la cooperación regional basada en el intercambio o transferencia de tecnologías, aplicaciones y programas informáticos; y a la promoción de la interoperabilidad en los sistemas de los gobiernos de la región sobre la base de estándares de integración. Por otro lado, se propone la interacción entre los servicios de las entidades de la administración pública nacional y local, y los ciudadanos a través de Internet, con el fin de

26 Como ejemplo se mencionan los centros de comunicación, capacitación, telecentros, estaciones de radio y televisión basadas en las comunidades (Cepal 2010a). 
obtener información relevante y realizar transacciones electrónicas vía portales electrónicos. De igual forma, se promueve la capacitación en el uso de las TIC a los funcionarios públicos, especialmente a los encargados de la toma de decisiones a nivel nacional, regional y local. Por último, como meta especial, se destaca la creación de mecanismos de estandarización y consolidación de la información georeferenciada, cuyo objetivo es proveer de herramientas adecuadas para la toma de decisiones de los gobiernos, el sector privado y las demás partes interesadas (Cepal 2010a).

- Capítulo 5. Sector productivo.- El quinto capítulo establece el fomento del acceso a los recursos y capacidades necesarias para la introducción de las TIC en los procesos productivos de las micro, pequeñas y medianas empresas, así como también para el desarrollo de empresas de tecnología e innovación. ${ }^{27}$ También se destaca el desarrollo de iniciativas para el acceso y uso de las TIC (incluyendo Internet, la telefonía fija y móvil, y los medios de comunicación tales como la radio y televisión) en el sector productivo. Por otra parte, se promueve la cooperación entre universidades, instituciones de formación profesional y el sector privado. Por último, se promueve la creación del teletrabajo, del trabajo móvil y de otras formas de trabajo por redes electrónicas; y las iniciativas del desarrollo de un portal regional para proporcionar información sobre buenas prácticas de uso de las TIC en las micro, pequeñas y medianas empresas (Cepal 2010a).

- Capítulo 6. Instrumentos de política y estrategias.- Finalmente, se proponen el fortalecimiento de las políticas nacionales para la sociedad de la información28 desde una perspectiva regional, incluyendo la participación de organismos públicos, la sociedad civil, el sector privado y académico; la creación o mantenimiento de un mecanismo de coordinación (punto focal) de las estrategias nacionales de la Sociedad de la Información; la elaboración de estudios comparativos sobre los impactos económicos y sociales de las TIC en las agendas políticas; el fomento de la inversión y los mecanismos de financiamiento; y el desarrollo de indicadores que den cuenta de los avances con el enfoque multiparticipativo en los procesos de políticas nacionales de desarrollo de la Sociedad de la Información (Cepal 2010a).

Adicionalmente, en este último capítulo se establecen temas como la solicitud al Observatorio para la Sociedad de la Información en América Latina y el Caribe, (Osilac), para continuar el monitoreo de actividades que permitan identificar las mejores prácticas e intercambio de experiencias con las TIC en la región;

27 Se refiere a empresas cuyo giro de negocio abarca básicamente la producción de hardware, el desarrollo de software, y la gestión de contenidos y servicios de telecomunicaciones (Cepal 2010a).

28 Siguiendo la misma línea de la Cepal, en el presente artículo se han utilizado indistintamente los términos referidos a Políticas TIC, Estrategias TIC o Agendas Digitales para referirse a las políticas públicas que buscan el desarrollo de la sociedad de la información de los estados (Cepal 2009). 
la promoción de acciones concretas de solidaridad y asistencia para facilitar el acceso a los beneficios de la sociedad de la información a los países de menor desarrollo y a los que presentan obstáculos en la implementación de estrategias nacionales con las TIC; el apoyo para que los diversos actores de la región progresen en el cumplimiento de los principios acordados en la CMSI; y el fomento de intercambio de experiencias y visiones de distintos actores sociales, regionales y nacionales sobre la relación entre género y TIC (Cepal 2010a).

4. Aproximación a las bases de una estrategia tecnológica: alineamiento del plan de acción regional eLAC al marco de acción de Hyogo

En base a los propósitos y contenidos expuestos acerca del Marco de Acción de Hyogo y el Plan de Acción Regional eLAC se determina una importante convergencia en la que ambos esfuerzos buscan ampliar las oportunidades de las personas para que a través del desarrollo de sus capacidades y libertades puedan conseguir un determinado bienestar y calidad de vida. Esta búsqueda del desarrollo humano se traduce en la consecución de los objetivos de desarrollo del milenio acordados internacionalmente para el periodo 2000-2015. Según esto, ambos marcos se encuentran orientados a contribuir con dichos objetivos por medio de la reducción del riesgo de desastres y la inclusión económica y social en la sociedad de la información.

Otro punto importante que sustenta esta relación es que tanto el MAH como el eLAC comparten lineamientos similares como la definición de los actores involucrados para su implementación: gobiernos, organismos regionales e internacionales, sociedad civil, sector privado y comunidad científica; el establecimiento de estrategias institucionales: políticas de reducción del riesgo y estrategias digitales, las cuales deben ser integradas en los planes de desarrollo nacionales bajo marcos normativos adecuados; la formación de plataformas nacionales y puntos focales encargados de definir y ejecutar las políticas, planes, estrategias y programas relacionados con ambos esfuerzos; el marco conceptual en el que se fundamentan, la reducción del riesgo de desastres y la sociedad de la información; el carácter transversal en las áreas claves del desarrollo: salud, educación, vivienda e infraestructura, servicios públicos, medioambiente, sector productivo, etcétera; la focalización de las estructuras sociales más vulnerables, incorporando la perspectiva de género y los temas culturales; el compromiso y voluntad política del gobierno y los grupos multisectoriales como factor trascendental para lograr un resultado satisfactorio; entre otros.

De igual manera, ambos modelos reconocen a la gestión de la información como un proceso crucial para el cumplimiento satisfactorio de sus objetivos. Mientras que el MAH establece dentro de sus prioridades de acción la sistematización de los 
procesos concernientes a la gestión del riesgo y el fortalecimiento de los sistemas de comunicación para las alertas tempranas y la respuesta ante desastres; el Plan de Acción Regional eLAC promueve el uso de las TIC para la gestión de los desastres en las fases de prevención, mitigación, preparación y respuesta, y establece además una serie de lineamientos que fomentan la interconexión de plataformas regionales, y el cumplimiento de estándares y regulaciones internacionales en materia de desastres. En vista de que el uso y acceso a la información son vitales para los propósitos de ambos modelos, las TIC son consideradas herramientas clave para apoyar su cumplimiento.

Es en base a esta convergencia que se considera factible y sinérgico la canalización de los esfuerzos propuestos en las metas del Plan de Acción Regional eLAC hacia la consecución de los objetivos y prioridades de acción del MAH. De esta manera, se sustenta el uso de las TIC como instrumentos estratégicos para la optimización de las políticas y acciones orientadas a la reducción del riesgo de desastres. Sin embargo, para lograr este objetivo es prioritario desarrollar las bases de una estrategia tecnológica que establezca los lineamientos necesarios para reorientar adecuadamente la gestión de la tecnología a la reducción del riesgo a nivel institucional y normativo. ${ }^{29}$ En ese sentido, el cuadro 3 muestra un esquema general de cómo esta estrategia tecnológica se alinea con la consecución del MAH a través de las metas del Plan de Acción Regional eLAC y el uso de las TIC.

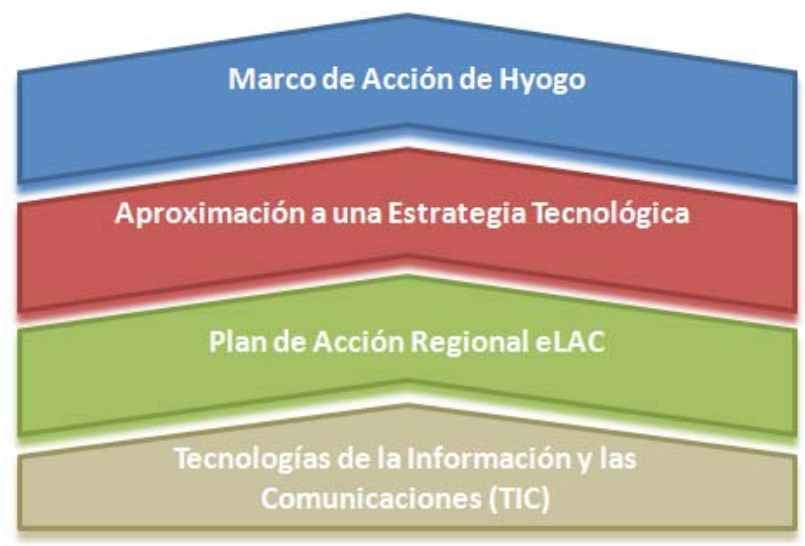

Cuadro 3. Consecución del MAH a través de la aproximación de una estrategia tecnológica que canaliza las metas del eLAC y el uso de las TIC.

Elaboración propia.

29 No se descarta que existan importantes propuestas similares a la aproximación de esta estrategia tecnológica las cuales posiblemente han sido desarrolladas e implementadas por entidades públicas especializadas, instituciones científicas y académicas, organismos internacionales y regionales, u organizaciones de la sociedad civil. Sin embargo, no es el propósito de este trabajo citarlas ni desarrollar su contenido. 
Cabe señalar que a nivel operacional, entre algunas de las TIC aplicadas mayormente en los procesos de la gestión del riesgo de desastres se encuentran la radio y la televisión (analógica y digital), las redes de telefonía móvil y fija, los mensajes de texto (Short Message Service, SMS), las redes y equipos de telecomunicación satelital; la teledetección y procesamiento de imágenes satelitales; internet y correo electrónico; las radios amateurs o radioaficionados; los sistemas de información geográfica (Geographic Information System, GIS), los sistemas de posicionamiento global (Global Positioning System, GPS), la gestión de sensores remotos (Remote Sensing); entre otras tecnologías importantes ${ }^{30}$ (Wattegama 2007).

Una vez definidos el alcance y el propósito de las bases de esta estrategia tecnológica, se procede a desarrollar sus principales lineamientos, los cuales se fundamentan en el objetivo general TIC, los objetivos estratégicos TIC y las prioridades orientadas a la gestión de la tecnología. Cabe destacar que todos estos elementos se encuentran agrupados en dos niveles estratégicos.

\subsection{Primer nivel estratégico}

En este primer plano se especifica la consecución del resultado esperado y los tres objetivos estratégicos del MAH a través del alineamiento de los objetivos TIC establecidos en la estrategia tecnológica. Si bien este alcance se asemeja a la realización de un Planeamiento Estratégico de Tecnologías de la Información (PETI), este estudio solo representa una aproximación teórica, la cual no intenta establecer un análisis o solución de un caso real. Asimismo, es importante mencionar que este nivel tiene como fin principal el cumplimiento de los objetivos de desarrollo del milenio a través de la implementación del Marco de Acción de Hyogo y los principios y metas de la Cumbre Mundial sobre la Sociedad de la Información, siendo el horizonte final el año 2015. El Cuadro 4 muestra en detalle el primer nivel estratégico.

Sobre esta base, en un primer subnivel, el objetivo general TIC de la estrategia tecnológica debe estar orientado al soporte y a la optimización de los procesos inherentes a la reducción del riesgo de desastres dentro del Marco de Acción de Hyogo, cuyo fin principal es la reducción de las pérdidas humanas, económicas y medioambientales ocasionadas por los desastres.

30 El documento ICT for Disaster Management hace una reseña sobre qué tecnologías deben ser utilizadas para cada fase de la gestión de desastres: reducción del riesgo, prevención, preparación, respuesta, recuperación y mitigación (Wattegama 2007). Asimismo, otra referencia importante es el documento ICT for Disaster Risk Reduction, en el cual se explica el rol importante que tienen las TIC en las fases de la reducción de riesgo y cuál ha sido su aplicación específica en los desastres ocurridos en China, Sri Lanka y Haití en 2010 (Apcict 2010). 


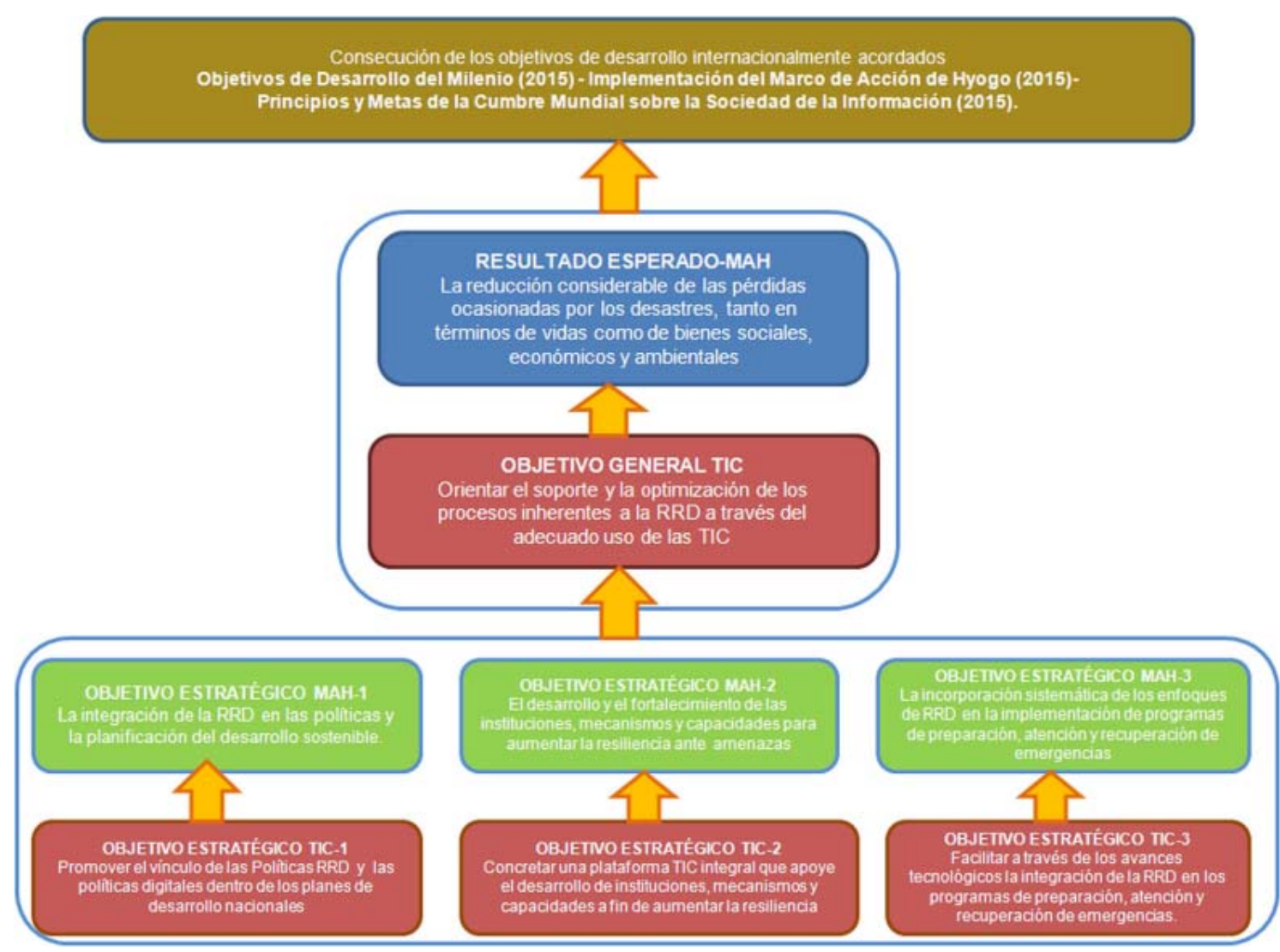

Cuadro 4. Primer nivel estratégico, alineamiento de los objetivos TIC definidos en la estrategia tecnológica para la consecución de los objetivos del MAH

Elaboración propia.

Considerando un segundo subnivel, la estrategia define tres objetivos estratégicos TIC que van alineados con los tres objetivos estratégicos definidos por el MAH. A continuación se realiza su descripción:

- Primer objetivo estratégico TIC: Establecer un vínculo entre las políticas sobre la reducción del riesgo de desastres y las políticas digitales definidas en los planes de desarrollo nacionales.

Esto da como resultado la posibilidad de consolidar sinergias en puntos específicos de ambas políticas, incrementado el énfasis en el uso de las TIC como instrumentos para potenciar la reducción del riesgo.

- Segundo objetivo estratégico TIC: Implementar una plataforma tecnológica integral que fomente el desarrollo y fortalecimiento de las instituciones, mecanismos y capacidades a fin de aumentar la resiliencia de las comunidades y países. 
Se considera a esta plataforma como el conjunto de acciones basadas en el uso de las TIC que, canalizadas a través del avance de las metas del Plan de Acción Regional eLAC, contribuyan al aumento de la resiliencia.

- Tercer objetivo estratégico TIC: Facilitar, a través de las tecnologías, la integración de la reducción del riesgo de desastres en los programas de preparación, respuesta y recuperación ante desastres.

Debido a que la gestión de la información representa un factor crítico para estos procesos, es necesario contar con un adecuado soporte tecnológico con el fin de garantizar el acceso y uso de este importante recurso.

\subsection{Segundo nivel estratégico}

En el segundo nivel se establece el ámbito en donde se reorientan los avances y resultados de las metas del Plan de Acción Regional eLAC hacia la mejora de la implementación de las prioridades de acción del Marco de Acción de Hyogo ${ }^{31}$ Cabe indicar nuevamente que para este estudio se han tomado como referencia las metas del Plan eLAC2010, el cual representó la segunda etapa de la estrategia regional eLAC para el periodo 2008-2010. De esta manera, con el fin de lograr este encauzamiento de esfuerzos, la estrategia tecnológica define cinco prioridades TIC, las cuales se alinean directamente con las cinco prioridades de acción del MAH.

El cuadro 5 muestra cómo las TIC representan la base tecnológica para el cumplimiento de las metas del Plan eLAC2010 agrupadas en seis capítulos, y cómo las prioridades TIC, a través de la aproximación de la estrategia tecnológica, se alinean hacia la consecución de las cinco prioridades del MAH. Asimismo, es importante que esta dinámica incorpore los temas transversales referidos a la perspectiva de género, la participación comunitaria, el desarrollo de capacidades y la transferencia tecnológica. De igual manera, se destaca la vital participación de los estados, organizaciones e instituciones regionales y organismos internacionales, incluyendo la Estrategia Internacional para la Reducción del Desastres y a la Comisión Económica para América Latina y el Caribe.

31 Cabe señalar que no todas las metas del Plan de Acción Regional eLAC pueden agregar valor a la Estrategia Tecnológica respecto a su apoyo en la implementación del Marco de Acción de Hyogo. Sin embargo, su contribución al desarrollo de la sociedad de la información incide de manera indirecta y fortalece las metas que sí le agregan valor. 


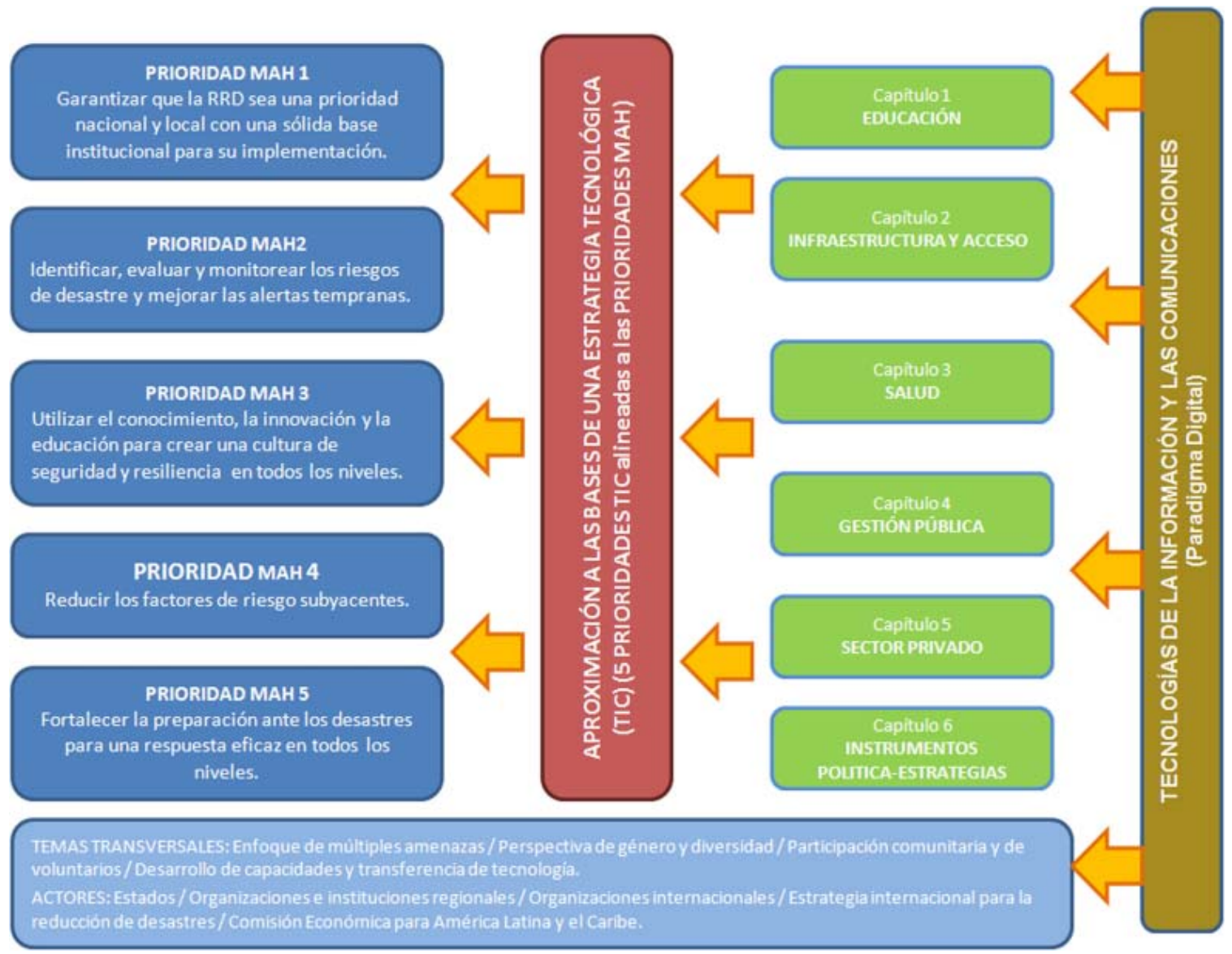

Cuadro 5. Segundo nivel estratégico, canalización de las metas del Plan eLAC2010, agrupadas en seis capítulos, con las cinco prioridades TIC de la Estrategia Tecnológica, para la consecución de las prioridades de acción del MAH.

Elaboración propia.

Con el fin de profundizar en la dinámica de este segundo nivel estratégico, se describen a continuación cada una de las cinco prioridades TIC de la estrategia tecnológica, las cuales se encuentran asociadas a metas específicas del Plan eLAC2010:

- Prioridad TIC 1: Garantizar la institucionalización de la estrategia digital32 para el desarrollo de la sociedad de la información a nivel nacional y local, e incluirla como apoyo para la Plataforma Nacional sobre la Reducción del Riesgo de Desastres. 
Se destaca la importancia de fortalecer el marco político y legal de la estrategia digital con el fin de establecer un vínculo institucional con el marco de la reducción del riesgo de desastres. De esta manera se crea una sólida oportunidad para alinear estrategias específicas TIC con la consecución de la reducción del riesgo en los ámbitos regional, nacional y local.

La Plataforma Nacional sobre la Reducción del Riesgo de Desastres, ${ }^{33}$ la cual está constituida por múltiples grupos sectoriales enfocados a la reducción de desastres, debe incluir necesariamente la participación de la entidad de coordinación que dirige la estrategia digital de un país. Se recomienda que las políticas, planes y estrategias de reducción del riesgo cuenten con el planeamiento y apoyo tecnológico necesario con el fin de facilitar su planificación, difusión y ejecución. Estas coordinaciones deben darse en los espacios de diálogo promovidos por los grupos interesados.

Del mismo modo, a través de la institucionalización de la estrategia digital se requiere contar con líneas presupuestarias dedicadas y mecanismos de financiación para el desarrollo y uso de las TIC que permitan potenciar las políticas y la ejecución de las acciones del Marco de Acción de Hyogo. Un aspecto importante es promover el empoderamiento y el desarrollo de las capacidades de los gobiernos o instituciones locales para que a través de los recursos asignados puedan realizar proyectos tecnológicos orientados a la reducción del riesgo en sus ámbitos geográficos en conjunto con las instituciones especializadas.

La gestión del punto focal nacional designado por la Plataforma Nacional sobre la Reducción del Riesgo de Desastres debe estar soportada por un sistema de información integrado ${ }^{34}$ que facilite la coordinación y ejecución de todas

32 La estrategia digital representa un conjunto de políticas, objetivos y lineamientos que establece un país con el fin de lograr su desarrollo en la sociedad de la información. En la región, esta estrategia es definida bajo el marco del Plan de Acción Regional eLAC. Tomando como referencia el caso del Perú, La Agenda Digital Peruana representa la estrategia digital del país, la cual fue establecida por la Comisión Multisectorial para el seguimiento y evaluación del Plan de Desarrollo de la Sociedad de la Información en el Perú (Codesi), y es actualmente dirigida por la Oficina Nacional de Gobierno Electrónico e Informática (Ongei).

33 En el caso peruano, la Plataforma Nacional sobre la Reducción del Riesgo de Desastres está representada por el Comité Multisectorial de Prevención y Atención de Desastres, encabezado por la Presidencia del Consejo de Ministros. Asimismo, el Instituto Nacional de Defensa Civil (Indeci), actúa como punto focal nacional dentro de las definiciones del Marco de Acción de Hyogo.

34 Este sistema de información integral representa un modelo teórico-genérico que tiene como fin reforzar y adaptarse a los lineamientos establecidos por la estrategia tecnológica. A modo de comparación, este sistema puede aproximarse al modelo colaborativo Cyberinfrastructure for Disaster Management propuesto en el trabajo A comprehensive conceptual model for disaster management (Asghar et al. 2006). Por otro lado, esta solución tecnológica también se refiere a un conjunto de procesos, personas, recursos, políticas, capacidades, aplicaciones a medida, software, bases de datos, componentes de hardware y sistemas de comunicación que trabajan de manera integrada hacia la consecución del objetivo de reducir los desastres. 
las estrategias y procesos relacionados con la reducción del riesgo. Sobre esta base, se propone la implementación o fortalecimiento, en el caso de que exista, de una solución que tenga como alcance los ámbitos nacional, provincial, comunitario y las zonas rurales de difícil acceso. ${ }^{35}$

Metas (M+) del Plan de Acción Regional eLAC2010 asociadas:

\section{(Capítulo 4 - Gestión pública)}

(M+37) Fortalecer los medios de intercambio sobre servicios de gobierno electrónico, desarrollando cooperación para el intercambio o transferencia de tecnologías, plataformas, aplicaciones y programas informáticos.

(M+38) Promover la interoperabilidad de sistemas de gobierno electrónico en la región, sobre la base de estándares y continuar la plataforma regional de interoperabilidad.

\section{(Capítulo 6 - Instrumentos de política y estrategias):}

$\mathbf{( M + 6 0 )}$ Promover y fortalecer las políticas nacionales para la sociedad de la información desde una perspectiva regional, incluyendo la coordinación con los distintos actores participantes.

(M+61) Confirmar y mantener una entidad o un mecanismo de coordinación de las estrategias digitales nacionales en todos los países para actuar como punto focal nacional.

(M+65) Contribuir a la concreción de recursos financieros para el desarrollo de sociedades de la información.

(M+66+67+68) Desarrollar indicadores, realizar trabajos estadísticos y monitorear el progreso de las etapas del Plan de Acción Regional a través de Osilac.

(M+69+70) Promover acciones de solidaridad y asistencia a los países de menor grado de desarrollo, a los estados insulares en desarrollo y a otros que enfrentan obstáculos en cuanto a la aplicación de estrategias digitales nacionales.

(M+78) Facilitar el diálogo y la coordinación de las diversas iniciativas regulatorias a nivel regional y local que pudieran favorecer la armonización del marco legal de la Sociedad de la Información en la región.

Fuente: Cepal (2010a).

35 Un esfuerzo importante que se debe considerar en el Perú es el Sistema Nacional de Información para la Prevención y Atención de Desastres (Sinpad), el cual es utilizado por las entidades que conforman el Sistema Nacional de Defensa Civil (Sinadeci) para la eficiente gestión del riesgo de desastres (Indeci 2011). 
- Prioridad TIC 2: Planificar e implementar las TIC para identificar, evaluar y monitorear los riesgos de desastres y potenciar las alertas tempranas.

La evaluación del riesgo es un paso fundamental para el desarrollo e implementación de políticas, planes y estrategias enfocados a la reducción del riesgo de desastres. Por ello, se requiere que el sistema integrado expuesto en la Prioridad TIC 1 permita la optimización y sistematización de los procesos de identificación, evaluación y monitoreo de los riesgos de desastre en todo el ámbito nacional. Estos procesos se basan en el análisis sistemático de las amenazas, vulnerabilidades y capacidades, y tienen como finalidad hallar y monitorear la distribución geográfica y sectorial del riesgo a través de los mapas de riesgo. Estas medidas guiarán y ayudarán a las autoridades responsables a tomar las decisiones adecuadas.

El sistema integrado deberá gestionar una base de evidencias sobre los riesgos de desastres, incluyendo registros históricos sobre amenazas, vulnerabilidades y pérdidas ocasionadas por los desastres. Para esto, es preciso contar con procesos que recopilen, desarrollen y actualicen la información crítica en tiempo real, lo cual es importante debido a la naturaleza cambiante del riesgo.

Asimismo, es necesario la optimización e integración de los sistemas de alerta temprana al sistema propuesto. Esta integración permite el acceso y uso de información crítica disponible con el fin de promover el desarrollo de capacidades de respuesta, los servicios de monitoreo de amenazas y alertas, la difusión y comunicación sobre riesgos y alertas y el conocimiento del riesgo. ${ }^{36}$ Cabe señalar que es necesario contar con un sólido sistema de comunicaciones a nivel nacional que permita difundir y comunicar por distintos medios la información real sobre riesgos y alertas tempranas a todos los sectores de la población. ${ }^{37}$

Se destaca la promoción de alianzas y asociaciones con organizaciones regionales e internacionales especializadas en la evaluación, monitoreo y difusión de información sobre amenazas naturales, biológicas y tecnológicas. El intercambio de información debe estar basado en un estándar internacional que garantice su interoperabilidad para cualquier sistema. Asimismo, es importante la integración de las plataformas de instituciones que manejan datos socioeconómicos, demográficos, inventarios de infraestructuras, ordenamiento territorial, y otros, para su uso en el proceso de evaluación de riesgos.

36 Estos cuatro elementos conforman los sistemas de alerta temprana centrados en las personas (EIRD 2007a).

37 Es imprescindible adaptar los sistemas de comunicación de alerta temprana a las necesidades de cada comunidad. Por ejemplo, se pueden utilizar la televisión, la radio; así como el sonido de sirenas, las banderas de alerta o los mensajes de texto (EIRD 2007a). 


\section{Metas (M+) del Plan de Acción Regional eLAC2010 asociadas:}

(Capítulo 2 - Infraestructura y acceso):

(M+11) Promover el acceso a las TIC, orientando a la real inserción social, educativa, cultural y económica a todos los sectores sociales, sobre todo a los más vulnerables.

(M+12) Desarrollar iniciativas de redes basadas en comunidades tales como centros de comunicación, telecentros, capacitación, estaciones radio y televisión basadas en comunidades. $(\mathbf{M}+\mathbf{1 6 + 1 7})$ Brindar cobertura a través de una red confiable, preferiblemente de alta capacidad, a la población en áreas urbanas y rurales.

$\mathbf{( M + 1 8 )}$ Incrementar el número de centros de acceso a las TIC en las comunidades, incluidas las bibliotecas y otras instalaciones.

$\mathbf{( M + 2 6 )}$ Fortalecer e interconectar las plataformas regionales para la gestión electrónica de procesos sobre desastres, promoviendo la colaboración de estos sistemas en base a estándares y acuerdos internacionales. ${ }^{38}$

\section{(Capítulo 4 - Gestión pública):}

(M+48) Promover la creación de mecanismos de estandarización y consolidación de la información georreferenciada, con el objetivo de que el gobierno, el sector privado y demás partes interesadas cuenten con herramientas útiles para la toma decisiones.

Fuente: Cepal (2010a).

- Prioridad TIC 3: Promover a través de las TIC la difusión del conocimiento, la innovación y la educación con el fin de crear una cultura de seguridad y resiliencia.

La estrategia de generar una cultura de seguridad y resiliencia requiere el uso de medios de comunicación y herramientas tecnológicas que garanticen la publicación y difusión de campañas orientadas a la sensibilización y concientización de las personas acerca de la reducción del riesgo. La promoción del acceso universal a estas tecnologías es un factor crítico de éxito ya que permite a las campañas tener un mayor alcance en torno a toda la población.

Un tema importante dentro del proceso de concientización es la incorporación de la reducción del riesgo de desastres en los sistemas educativos nacionales y en las comunidades de investigación científica. Para lograr esta tarea, se requiere facilitar el acceso a redes educativas, incluyendo internet, plataformas de educación a distancia y herramientas tecnológicas que permitan a las escuelas, centros de educación superior e instituciones científicas acceder, investigar e impartir materias sobre la reducción de desastres.

Además, cabe señalar que como parte de las capacitaciones en los sectores claves del desarrollo, es necesario brindar formación en el uso de herramientas TIC no solo en el nivel del usuario sino también en el ámbito especializado res-

38 Entre los organismos que fueron responsables de llevar a cabo esta meta se encuentran el Caribbean Disaster Emergency Management Agency (Cdema, antes Cedera), el Centro Regional de Información sobre Desastres de América Latina y el Caribe (CRID), la Estrategia Internacional para la Reducción de Desastres (EIRD) y la Unión Internacional de Telecomunicaciones (UIT) (Cepal 2010a). 
pecto de los sistemas relacionados a la reducción del riesgo de desastres. Esta capacitación debe estar dirigida a los tomadores de decisiones, los funcionarios públicos, los profesionales, los técnicos y los gestores en el tema de desastres.

Por último, se propone implementar y mejorar los centros de información nacionales sobre amenazas, vulnerabilidades, riesgos y desastres, bajo una plataforma tecnológica integrada, confiable y disponible. Esta implementación facilita la recopilación, difusión y utilización de la información crítica que va a ser utilizada por los gobiernos, las instituciones especializadas, la comunidad científica, los profesionales y las comunidades. Esta solución necesita estar integrada con la solución integral.

Metas (M+) del Plan de Acción Regional eLAC2010 asociadas:

\section{(Capítulo 1 - Educación / Capítulo 6 - Gestión pública):}

$\mathbf{( M + 1 )}$ Desarrollar programas de estudio que contemplen el manejo de datos, información y conocimiento.

$\mathbf{( M + 2 )}$ Elaborar estudios anuales sobre el impacto del uso de las TIC en el sistema educativo.

$\mathbf{( M + 3 ) ~ G a r a n t i z a r ~ e l ~ a c c e s o ~ a ~ i n t e r n e t ~ ( b a n d a ~ a n c h a ) ~ a ~ l a s ~ i n s t i t u c i o n e s ~ d e ~ e n s e n ̃ a n z a ~}$ pública.

$\mathbf{( M + 4 + 5 + 6 + 4 1 + 4 2 )}$ Capacitar a alumnos, profesores, funcionarios públicos y responsables de toma de decisión en el uso de herramientas TIC.

$\mathbf{( M + 7 + 8 + 9 + 1 0 )}$ Garantizar el uso de herramientas TIC orientadas a programas educativos, tales como portales, aplicación de contenidos, Web 2.0 y realidad virtual.

\section{(Capítulo 2 - Infraestructura y acceso):}

$\mathbf{( M + 1 1 )}$ Promover el acceso a las TIC, orientándose a la real inserción social, educativa, cultural y económica, a todos los sectores sociales, sobre todo a los más vulnerables. (M+12) Desarrollar iniciativas de redes basadas en comunidades tales como centros de comunicación, telecentros, capacitación, estaciones de radio y televisión basadas en comunidades.

$\mathbf{( M + 1 3 )}$ Apoyar iniciativas regionales y subregionales que aprovechan las economías de escala para atender a los sectores marginados por los avances tecnológicos (@LIS, RedCLARA, C@ribNET, entre otros).

$\mathbf{( M + 1 6 + 1 7 ) ~ B r i n d a r ~ c o b e r t u r a ~ a ~ t r a v e ́ s ~ d e ~ u n a ~ r e d ~ c o n f i a b l e , ~ p r e f e r i b l e m e n t e ~ d e ~ a l t a ~}$ capacidad, a la población en áreas urbanas y rurales.

$\mathbf{( M + 1 8 ) ~ I n c r e m e n t a r ~ e l ~ n u ́ m e r o ~ d e ~ c e n t r o s ~ d e ~ a c c e s o ~ a ~ l a s ~ T I C ~ e n ~ l a s ~ c o m u n i d a d e s , ~ i n - ~}$ cluidas las bibliotecas y otras instalaciones.

$\mathbf{( M + 1 9 )}$ Conectar a los centros de investigación y educación a las redes avanzadas de datos para la investigación.

$\mathbf{( M + 2 1 + 2 2 )}$ Elaborar estudios bienales sobre la estructura de precios de los servicios de telecomunicaciones de la telefonía fija, móvil e internet; e impulsar la creación de canastas de servicios digitales a precios especiales orientados a los sectores más vulnerables. $\mathbf{( M + 2 3 )}$ Revisar la funcionalidad, el diseño y el objeto de los fondos de acceso universal a las TIC

Fuente: Cepal (2010a). 
- Prioridad TIC 4: Garantizar a través de las TIC la reducción de los factores de riesgos subyacentes.

Para el sector ambiental, se propone la implementación de sistemas de información que apoyen el desarrollo de las estrategias de gestión medioambiental y uso sostenible de los recursos naturales. A través de estos sistemas se pretende identificar y monitorear las zonas afectadas por la degradación ambiental; gestionar el inventario de recursos naturales y apoyar la ejecución de estudios de impacto ambiental (EIA).

Como medida complementaria a los programas sociales enfocados al aumento de la resiliencia de las personas en situación de pobreza y vulnerabilidad, se requiere facilitar el acceso universal a las TIC como medio para contribuir al incremento del empoderamiento; al acceso a la información sobre amenazas, riesgos y alertas tempranas; y al acercamiento de las autoridades locales y gobiernos con dichos grupos vulnerables, sobre todo para las zonas urbanas y rurales con difícil acceso.

También se propone la utilización de herramientas TIC especializadas para el apoyo y optimización de los procesos de planificación urbana y ordenamiento territorial, las cuales se orientan a la reducción de riesgos. Estos procesos se basan en la información sobre estudios de planificación, mapas geográficos, análisis de impacto ambiental y amenazas, características sobre suelos y tierras, entre otros temas.

Considerando el ámbito del sector privado, especialmente el rubro tecnológico, se requiere estimular a las empresas de desarrollo de soluciones y servicios TIC para que diseñen, produzcan y brinden servicios de tecnología orientados a la reducción del riesgo de desastres. Asimismo, a través del uso de las tecnologías, se debe apoyar el aseguramiento de la continuidad y resiliencia de los sectores de producción y servicios ante situaciones de emergencia o desastre. En base a esto, es necesario establecer alianzas entre el gobierno y el sector privado, especialmente a través de la intervención del sector de telecomunicaciones, el cual debe definir las bases y el marco regulatorio correspondiente.

Los sistemas de comunicaciones representan una de las infraestructuras más críticas que deben asegurar los estados durante el continuo proceso de la reducción del riesgo, sobre todo en situaciones de desastre y la posterior recuperación. Esta infraestructura debe estar disponible en todo momento ya que interconecta hospitales; centros de información, operaciones y emergencias; edificios de servicios gubernamentales; instalaciones de refugios y almacenes, y rutas de transporte. Además, estos representan la base tecnológica que permite el funcionamiento de los sistemas que gestionan información crítica sobre los riesgos y los desastres, en este caso el sistema integrado expuesto. Para esto, se necesita desarrollar iniciativas de implementación de redes de contingen- 
cia o respaldo a través distintas tecnologías de comunicación disponibles. Esto debe implementarse de manera conjunta con el sector privado, proveedores de telecomunicaciones, a través de acuerdos de niveles de servicio y alianzas público-privadas.

Del mismo modo, se destaca la importancia de considerar la disponibilidad de los sistemas de información y comunicaciones como apoyo tecnológico en la planificación y ejecución de los planes de recuperación y contingencia posdesastre. Estos sistemas deben facilitar la coordinación entre los distintos niveles de gobierno, las agencias especializadas, la sociedad civil y el sector privado en las fases de respuesta y recuperación posdesastre. Por otra parte, los planes deben especificar procedimientos para reestablecer los servicios de información y comunicaciones afectados durante el desastre.

Metas (M+) del Plan de Acción Regional eLAC2010 asociadas:

\section{(Capítulo 2 - Infraestructura y acceso):}

$\mathbf{( M + 1 4 )}$ Impulsar el desarrollo de la infraestructura en cada país y la región con el fin de mejorar la calidad y estabilidad de la red, y reducir los costos de acceso.

$\mathbf{g}(\mathbf{M}+\mathbf{2 4})$ Fomentar el desarrollo de infraestructura para satisfacer las necesidades de flujo de tráfico, la continuidad de los servicios de interconexión y el intercambio de experiencias sobre el impacto del proceso de convergencia de las TIC.

\section{(Capítulo 3 - Salud):}

$\mathbf{( M + 2 9 + 3 3 ) ~ P r o m o v e r ~ l a ~ i n t e g r a c i o ́ n ~ d e ~ l a s ~ T I C , ~ e l ~ d i a g n o ́ s t i c o ~ s i t u a c i o n a l , ~ l a ~ i d e n t i f i c a - ~}$ ción de desafíos regionales y las mejores prácticas en el ámbito del sector salud.

\section{(Capítulo 4 - Gestión pública):}

(M+39+40) Garantizar que los gobiernos locales interactúen con los ciudadanos y con otras ramas de la administración pública nacional y local, incluyendo la realización de transacciones electrónicas.

\section{(Capítulo 5 - Sector productivo):}

$\mathbf{( M + 4 9 + 5 0 )}$ Facilitar el acceso a los recursos y capacidades para la introducción de las TIC en los procesos productivos de las micro, pequeñas y medianas empresas; así como también para el desarrollo de empresas de tecnología, estimulando la innovación de productos y servicios tecnológicos.

$\mathbf{M + 5 7 )}$ Promover la creación de teletrabajo, trabajo móvil y otras formas de trabajo por redes electrónicas, sobre todo para los grupos más vulnerables e incluidas las personas con discapacidad.

\section{(Capítulo 6 - Instrumentos de políticas y estrategias):}

$(\mathbf{M + 7 3})$ Facilitar el intercambio de experiencias y visiones de distintos actores sociales sobre la relación entre género y TIC. 
- Prioridad TIC 5: Fortalecer a través de las TIC la preparación en caso de desastres con el fin de brindar una respuesta eficaz.

Frente a la necesidad de fortalecer la preparación ante los desastres, existe el requerimiento de contar con un proceso sistemático que permita el desarrollo de los planes de preparación y contingencia. Por otro lado, ante la ocurrencia del desastre, la plataforma tecnológica debe facilitar la coordinación entre todos los actores relevantes, la gestión de la información crítica, la gestión de los recursos y finanzas, la evaluación de daños y el análisis de necesidades, la difusión de alertas tempranas e información relevante para los afectados y la programación de operaciones en el terreno.

Dentro del proceso de preparación, se propone la planificación de evaluaciones periódicas y mejoras en las capacidades del sistema integrado, los centros de información, los sistemas de alerta temprana y la infraestructura de comunicaciones. El propósito de esto es garantizar la disponibilidad y confiabilidad tecnológica necesaria para apoyar la gestión de emergencias ante cualquier situación de desastre.

Asimismo, se requiere incentivar la coordinación, cooperación y asistencia técnica entre entidades nacionales, e internacionales que asesoren, informen y brinden las mejores prácticas en el uso de herramientas tecnológicas con el fin de afrontar situaciones de desastres, tanto en las fases de respuesta como en las fases de preparación y recuperación.

Metas (M+) del Plan de Acción Regional eLAC2010 asociadas:

\section{(Capítulo 2 - Infraestructura y acceso):}

(M+14) Impulsar el desarrollo de la infraestructura en cada país y la región con el fin de mejorar la calidad y estabilidad de la red y reducir los costos de acceso.

$\mathbf{( M + 2 4 )}$ Fomentar el desarrollo de infraestructura para satisfacer las necesidades de flujo de tráfico, la continuidad de los servicios de interconexión y el intercambio de experiencias sobre el impacto del proceso de convergencia TIC.

$\mathbf{( M + 2 8 )}$ Promover sistemas de gestión de emergencias, incluyendo el manejo de la respuesta, los recursos y la base de datos de los damnificados.

$\mathbf{( M + 2 7 )}$ Fomentar acuerdos en los cuales países vecinos provean infraestructura de respaldo para los sistemas de gestión de emergencias en caso de desastres.

\section{(Capítulo 6 - Instrumentos de políticas y estrategias):}

$\mathbf{( M + 8 0 )}$ Invitar a la adopción del Convenio de Tampere ${ }^{39}$ sobre el suministro de recursos de telecomunicaciones para la mitigación y respuesta de emergencia ante desastres a los países que no lo han ratificado aún.

Fuente: Cepal (2010a)

39 Un claro ejemplo es el Convenio de Tampere, el cual representa un tratado internacional que hace referencia al suministro de recursos de telecomunicaciones para la mitigación y las operaciones de socorro en casos de desastres. Este convenio pide a los estados que proporcionen asistencia de manera rápida en materia de 


\section{Conclusiones}

La reducción del riesgo de desastres representa un tema prioritario en la agenda global ante la constante ola de desastres que interrumpen considerablemente la continuidad del proceso de desarrollo de las naciones. Considerando el panorama actual de América Latina y el Caribe, donde la constante ocurrencia de desastres de gran magnitud es ocasionada por la combinación de las múltiples amenazas y las acentuadas vulnerabilidades socioeconómicas, políticas y ambientales existentes, se evidencia la necesidad de contar con todos los instrumentos disponibles que permitan fortalecer la implementación de políticas, planes y estrategias orientadas a la reducción de los desastres.

Si bien las tecnologías de la información y las comunicaciones tienen el poder de revolucionar las industrias y transformar la manera en que se realizan los negocios, estas también representan instrumentos estratégicos que añaden valor y soportan las áreas claves del desarrollo. Sobre esta base, es consecuente definir una serie de lineamientos que guíen a través del uso de las TIC la optimización de los procesos orientados a la reducción del riesgo. Por consiguiente, se considera prioritaria la definición de las bases de una estrategia tecnológica que permita canalizar los esfuerzos propuestos en las metas del Plan de Acción Regional eLAC hacia la consecución de los objetivos y prioridades de acción del Marco de Acción de Hyogo. Cabe señalar que esta aproximación estratégica representa solo una iniciativa distinta dentro de una totalidad de esfuerzos encaminados a la manera de utilizar las TIC para la reducir los desastres.

Mientras que el Marco de Acción de Hyogo representa el consenso internacional más importante sobre la reducción del riesgo de desastres en la actualidad el Plan de Acción Regional sobre la Sociedad de la Información constituye un acuerdo regional enfocado al desarrollo de las sociedades digitales de los países de Latinoamérica. A partir de su estudio, se evidencia que los propósitos y contenidos de ambos esfuerzos convergen de manera sinérgica en la consecución de los objetivos de desarrollo del milenio, teniendo en consideración las características y estructuras similares que comparten y el reconocimiento de la criticidad de la gestión de la información en los procesos de la reducción del riesgo. Es a partir de esta convergencia que se pone de manifiesto la factibilidad teórica de encaminar

telecomunicaciones para aliviar el impacto de los desastres, contemplando la instalación y puesta en marcha de los servicios de telecomunicaciones, y suprimiendo los obstáculos reglamentarios que impiden la utilización de los equipos para llevar a cabo las tareas de rescate. Asimismo, las bases para la adopción de este tratado fueron establecidas en la Conferencia Intergubernamental de Urgencia (ICET-98) celebrada en enero de 1998 en Tampere, Finlandia. Posteriormente, este entró en vigor en enero del 2005 tras la ratificación de treinta países (ITU 2010a). 
y articular a través de los lineamientos de esta estrategia tecnológica las metas del Plan de Acción Regional eLAC hacia el cumplimiento del Marco de Acción de Hyogo.

Es entonces que dado el carácter institucional y normativo que significaría incorporar los lineamientos de esta estrategia en los planes y procesos de reducción de desastres de cualquier Estado, se considera fundamental contar con la voluntad y el compromiso político de las autoridades y grupos multisectoriales; sobre todo de aquellos sectores que son responsables de gestionar las políticas de reducción del riesgo, las estrategias digitales y las áreas claves del desarrollo. Sumado a esto, otro factor crítico que afectaría la viabilidad ${ }^{40}$ de esta propuesta es el grado de institucionalización del Marco de Acción de Hyogo y del Plan de Acción Regional eLAC en las políticas y planes de desarrollo de los países. En base a estas premisas, un escenario satisfactorio daría como resultado la disponibilidad de mecanismos institucionales que promoverían el uso de las TIC como instrumentos de apoyo estratégicos para la reducción del riesgo de desastres en los ámbitos nacional, local y comunitario. A partir de esta institucionalización se desplegarían los lineamientos operacionales y técnicos que definirían qué tecnología específica debería ser utilizada para cada realidad social o situación de desastre.

Adicionalmente, es importante que los gobiernos y grupos multisectoriales trabajen de manera conjunta y colaborativa con los organismos internacionales y regionales especializados, las instituciones científicas y académicas, las organizaciones de la sociedad civil, el sector privado y, en especial, con la Estrategia Internacional para la Reducción de Desastres (EIRD) y la Comisión Económica para América Latina y el Caribe (Cepal). Esto traería como resultado el intercambio de conocimientos y buenas prácticas, el aumento de las capacidades y recursos y una adecuada transferencia tecnológica con el fin de lograr la aplicación de las TIC en los procesos de la reducción del riesgo.

Un elemento crucial para la incorporación de esta estrategia es la focalización de los grupos sociales más vulnerables. De acuerdo con esto, es necesario universalizar el acceso y uso de las TIC en la medida que se reduzca la exclusión digital que sufren dichos colectivos. El avance en la inclusión digital permitiría el apoyo en la difusión de programas de prevención y sensibilización sobre el riesgo de desastres, y la toma de acciones apropiadas ante la ocurrencia de emergencias a niveles nacional, local y comunitario. Asimismo, se destaca que esta estrategia

40 Cabe señalar que esta viabilidad también depende mucho de la situación coyuntural de cada gobierno y de las prioridades definidas en sus respectivas políticas y planes de desarrollo. En ese sentido, la mayoría de países latinoamericanos destinan sus esfuerzos en la atención de los sectores de salud, vivienda, educación, agricultura, producción, etcétera; quedando la reducción del riesgo de desastres y el desarrollo de la sociedad de la información como áreas no tan prioritarias para atender. 
debe ser de carácter transversal en el sentido que promueva la participación comunitaria, la perspectiva de género, el desarrollo de capacidades humanas, el cuidado del medio ambiente y la transferencia tecnológica.

Asimismo, otro aspecto a considerar es la repercusión directa que tiene el desarrollo de la Sociedad de la Información sobre la aplicación de las TIC en los procesos de reducción de desastres. Esto significa que mientras más avanzado se encuentre un país respecto a su sociedad de la información mejor resultado tendrá esta estrategia tecnológica al enfocar y maximizar su apoyo en la implementación del Marco de Acción de Hyogo. De esta manera, el progreso de las metas del Plan de Acción Regional eLAC en sus tres etapas representa un factor clave para garantizar la disponibilidad de soluciones tecnológicas que puedan contribuir con la reducción del riesgo de desastres.

Finalmente, se evidencia la necesidad de que los lineamientos de esta estrategia tecnológica sean dinámicos en el tiempo y adaptables ante cualquier circunstancia. Para esto deben ser consideradas las constantes amenazas y vulnerabilidades en la región, y los acelerados cambios tecnológicos en el contexto de la sociedad de la información. En ese sentido, esta iniciativa debe ser coherente con las proyecciones a niveles global y regional del Marco de Acción de Hyogo y el Plan de Acción Regional eLAC, respectivamente, al año 2015 y a su continuación. De acuerdo con esto, la estrategia debe incorporar mecanismos de evaluación y mejora continua con el fin de adaptarse y ser consecuente con la coyuntura global.

\section{Siglas y semisiglas empleadas en este texto}

\begin{tabular}{|l|l|}
\hline Apcict & $\begin{array}{l}\text { Asian Pacific Training Centre for Information and Communication } \\
\text { Technology for Development }\end{array}$ \\
\hline Caprade & Comité Andino para la Prevención y Atención de los Desastres \\
\hline Cdema & Caribbean Disaster Emergency Management Agency \\
\hline Cepal & Comisión Económica para América Latina y el Caribe \\
\hline Cepredenac & $\begin{array}{l}\text { Centro de Coordinación para la Prevención de Desastres Naturales } \\
\text { en América Central }\end{array}$ \\
\hline CMDS & Cumbre Mundial sobre el Desarrollo Sostenible \\
\hline CMSI & Cumbre Mundial sobre la Sociedad de la Información \\
\hline CMRD & Conferencia Mundial sobre la Reducción de Desastres \\
\hline Codesi & $\begin{array}{l}\text { Comisión Multisectorial para el Seguimiento y Evaluación del Plan } \\
\text { de Desarrollo de la Sociedad de la Información en el Perú }\end{array}$ \\
\hline
\end{tabular}


Aproximación teórica a las bases de una estrategia tecnológica orientada a la reducción del riesgo de desastres

\begin{tabular}{|l|l|}
\hline CRED & Centre of Research on the Epidemiology of Disasters \\
\hline CRID & $\begin{array}{l}\text { Centro Regional de Información sobre Desastres de América Latina } \\
\text { y el Caribe }\end{array}$ \\
\hline DIRDN & Decenio Internacional para la Reducción de Desastres Naturales \\
\hline EM-DAT & Emergency Events Database \\
\hline EIRD & Estrategia Internacional para la Reducción de Desastres \\
\hline ICT & Information and Communications Technologies \\
\hline Indeci & Instituto Nacional de Defensa Civil \\
\hline ITU & International Telecommunications Union \\
\hline ISDR & International Strategy for Disaster Reduction \\
\hline MAH & Marco de Acción de Hyogo \\
\hline OCAH & $\begin{array}{l}\text { Oficina de las Naciones Unidas para la Coordinación de Asuntos } \\
\text { Humanitarios }\end{array}$ \\
\hline OCHA & Office for the Coordination of Humanitarian Affairs \\
\hline OMM & Organización Meteorológica Mundial \\
\hline ONGEI & Oficina Nacional de Gobierno Electrónico e Informática \\
\hline ONU & Organización de las Naciones Unidas \\
\hline Osilac & $\begin{array}{l}\text { Observatorio para la Sociedad de la Información en América Latina } \\
\text { y el Caribe }\end{array}$ \\
\hline PG/RRD & Plataforma Global para la Reducción del Riesgo de Desastres \\
\hline PNUMA & Programa de las Naciones Unidas para el Medio Ambiente \\
\hline PNUD & Programa de las Naciones Unidas para el Desarrollo \\
\hline RRD & Reducción del Riesgo de Desastres \\
\hline Sinadeci & Sistema Nacional de Defensa Civil \\
\hline Sinpad & $\begin{array}{l}\text { Sistema Nacional de Información para la Prevención y Atención de } \\
\text { Desastres }\end{array}$ \\
\hline TIC & Tecnologías de la Información y las Comunicaciones \\
\hline UNDP & United Nations Development Program \\
\hline UnesCO & Organización de las Naciones para la Educación, Ciencia y Cultura \\
\hline Unicef & United Nations Children's Fund \\
\hline UIT & Unión Internacional de Telecomunicaciones \\
\hline WSIS & World the Information Society \\
\hline
\end{tabular}




\section{Bibliografía}

Acevedo, Manuel; Chereguini, Estefanía; Hidalgo, Richard C.; Mataix, Carlos; Moreno, Ana y Eduardo Sánchez (2006). Guía para la integración de las TIC en la cooperación española. Madrid: Ministerio de Asuntos Exteriores y de Cooperación, Secretaría de Estado de Cooperación Internacional.

APCICT (2010). "ICT for Disaster Risk Reduction". ACPICT. http://www.unapcict.org/ ecohub/ict-for-disaster-risk-reduction-1 [Consulta: 15 de octubre del 2010).

Asghar, Sohail; Alahakoon, Damminda y Leoind Churilov (2006). "A comprehensive conceptual model for disaster management". Clayton school of Information Technology, Monash University. http://www.reliefweb.int/rw/lib.nsf/ db900SID/AMMF6RWDTG?OpenDocument [Consulta: 15 de octubre del 2010].

Castells, Manuel (1997). La era de la información: Economía, sociedad y cultura. Volumen I: La sociedad red. Madrid: Alianza Editorial.

Cepal (2011a)."Declaración de Lima". Tercera Conferencia Ministerial sobre la Sociedad de la Información en América Latina y el Caribe. Cepal. http://www.eclac.cl/ socinfo/noticias/documentosdetrabajo/3/41773/2010-876-eLAC-Declaracion_de_Lima.pdf [Consulta: 13 de junio del 2011).

-. (2011b)."PlandeAcciónsobrelaSociedaddelalnformaciónydelConocimiento de América Latina y el Caribe (eLAC2015)". Tercera Conferencia Ministerial sobre la Sociedad de la Información en América Latina y el Caribe. Cepal. http:// www.eclac.cl/socinfo/noticias/documentosdetrabajo/0/41770/2010-819eLAC-Plan_de_Accion.pdf [Consulta: 13 de junio del 2011].

- . (2010a). "Compromiso de San Salvador". Segunda Conferencia Ministerial sobre la Sociedad de la Información en América Latina y el Caribe. Cepal._http:// www.cepal.org/socinfo/noticias/noticias/2/32362/2008-1TICsCompromiso_de_San_Salvador.pdf [Consulta: 10 de septiembre del 2010].

- . (2010b). "Antecedentes políticos". Programa Sociedad de la Información. Cepal. http://www.cepal.org/cgibin/getprod.asp?xml=/socinfo/noticias/ paginas/2/32522/P32522.xml\&xsl=/socinfo/tpl/p18f-st.xsl\&base=/socinfo/ tpl/top-bottom.xsl [Consulta: 7 de septiembre del 2010].

-. $\quad$ (2010c)."eLAC2007:El primer Plan de Acción Regional (2005-2007)". Programa Sociedad de la Información. Cepal. http://www.cepal.org/cgi-bin/getprod. asp?xml=/socinfo/noticias/paginas/4/32534/P32534.xml\&xsl=/socinfo/tpl/ p18f-st.xsl\&base=/socinfo/tpl/top-bottom.xsl. [Consulta: 7 de septiembre del 2010]. 
-. (2010d). "Monitoreo del Plan elAC2010: Avances y desafíos de la Sociedad de la Información en América Latina y el Caribe". eLAC 2015. Documentos. http://www.eclac.cl/elac2015/documentos/ [Consulta: 8 de octubre del 2010].

- (2009). La Sociedad de la Información en América Latina y el Caribe: Desarrollo de las tecnologías y tecnologías para el desarrollo. Santiago de Chile: Cepal.

- . (2007). "Plan de Acción sobre la Sociedad de la Información de América Latina y el Caribe". Plan de Acción eLAC 2007. http://www.eclac.cl/socinfo/noticias/documentosdetrabajo/8/21678/eLAC_2007_Espanol.pdf [Consulta: 6 de octubre del 2010].

CMSI (2005). "Documentos finales: Ginebra 2003-Túnez 2005". Cumbre Mundial sobre la Sociedad de la Información. http://www.itu.int/wsis/outcome/ booklet-es.pdf [Consulta: 13 de septiembre del 2010].

CRED (2011). "Result for Country Profile". EM-DAT. http://www.emdat.be/resultcountry-profile [Consulta: 15 de junio del 2011].

EIRD (2011a). "Documento de debate para la tercera reunión de la Plataforma Mundial para la Reducción del Riesgo de Desastres y Conferencia Mundial sobre Reconstrucción". Plataforma Mundial para la Reducción del Riesgo de Desastres, Tercera Reunión, 2011. http://www.preventionweb.net/globalplatform/2011/documents/documents/DiscussionPaper_ES.pdf [Consulta: 16 de junio 2011].

- . (2011b). "OAS. Informe regional del progreso en la implementación del Marco de Acción de Hyogo (2009-2011)". PreventionWeb. http://www.preventionweb.net/files/19612_RegionalHFAprogress-OAS(2009-2011).pdf. [Consulta: 16 de junio 2011].

- . (2010). "Global Platform for Disaster Risk Reduction". PreventionWeb. http:// www.preventionweb.net/english/hyogo/GP/ [Consulta: 28 de septiembre del 2010].

-. (2009). "Documento de resultados finales: Resumen del Presidente sobre la segunda sesión de la Plataforma Global para la Reducción del Riesgo de Desastres". Plataforma Global para la Reducción del Riesgo.MAH. http://www. preventionweb.net/files/10750_ChairsSummaryGP2009FINALSpanish.pdf [Consulta: 28 de septiembre del 2010].

- - (2007a). De las palabras a la acción: Guía para la implementación del Marco de Acción de Hyogo 2005 -2015: Aumento de la resiliencia de las naciones y las comunidades ante los desastres. Ginebra: EIRD. 
- - (2007b). "Propuesta del Subsecretario General para Asuntos Humanitarios de fortalecer el sistema de la EIRD", PG/RRD, Primera sesión Ginebra, Suiza, http://www.preventionweb.net/globalplatform/2007/first-session/docs/ info_docs/GP_2007_inf2_Sp.pdf, (fecha de acceso: 02 de octubre 2010).

- - (2004). Vivir con el riesgo: Informe mundial sobre iniciativas para la reducción de desastres. Ginebra: EIRD.

Guha-Sapir, D.; Femke, Vos; Regina, Below y Sylvain Ponserre (2011). Annual Disaster Statistical Review 2010: The numbers and trends. Brussels: CRED.

Indeci (2011). "Sistema Nacional de Información para la Prevención y Atención de Desastres". Indeci. http://sinpad.indeci.gob.pe/PortalSINPAD/Default. aspx?ltemld=1 [Consulta: 18 de octubre del 2011].

ITU (2010a). "El Convenio de Tampere: Un tratado que salva vidas". ITU. http://www. itu.int/ITU-D/emergencytelecoms/tampere-es.html [Consulta: 18 de octubre del 2010].

ONU (2011). "Podemos erradicar la pobreza: Objetivos de Desarrollo del Milenio 2015". Portal de la labor del Sistema de las Naciones Unidas sobre los Objetivos de Desarrollo del Milenio. http://www.un.org/spanish/millenniumgoals/ [Consulta: 24 de junio del 2011].

- . (2008). "Estrategia Internacional para la Reducción de Desastres". Asamblea General, A/RES/62/192. http://www.unisdr.org/eng/about_isdr/basic_ docs/GA-resolution/a-res-62-192-spa.pdf [Consulta: 2 de septiembre del 2010].

-. (2005). "Informe de la Conferencia Mundial sobre la Reducción de Desastres: Kobe, Hyogo (Japón), 18 a 22 de enero de 2005". Conferencia Mundial sobre la Reducción de Desastres, A/CONF.206/6. http://www.unisdr.org/eng/hfa/ docs/final-report-wcdr-spanish.pdf [Consulta: 20 de junio del 2010].

PNUD (1990). "Informe sobre desarrollo humano 1990". Informes sobre desarrollo humano. http://hdr.undp.org/es/informes/mundial/idh1990/capitulos/espanol/ (Consulta: 24 de junio del 2011 ].

Wattegama, Chanuka (2007). ICT for Disaster Management. Thailand: UNDP, AsiaPacific Development Information Programme (APDIP)/Asian and Training Centre for Information Centre for Information and Communication Technology for Development (APCICT). 